\title{
Polarization measurements in neutral pion photoproduction
}

K. Wijesooriya, ${ }^{1, *}$ A. Afanasev, ${ }^{21,27}$ M. Amarian, ${ }^{12}$ K. Aniol, ${ }^{4}$ S. Becher, ${ }^{10}$ K. Benslama, ${ }^{24}$ L. Bimbot, ${ }^{23}$ P. Bosted, ${ }^{17}$ E. J. Brash, ${ }^{24}$ J. Calarco, ${ }^{19}$ Z. Chai, ${ }^{18}$ C. C. Chang, ${ }^{16}$ T. Chang, ${ }^{11}$ J. P. Chen, ${ }^{27}$ S. Choi ${ }^{26}$ E. Chudakov, ${ }^{27}$ S. Churchwell, ${ }^{7}$ D. Crovelli, ${ }^{25}$ S. Dieterich, ${ }^{25}$ S. Dumalski, ${ }^{24}$ D. Dutta, ${ }^{18}$ M. Epstein, ${ }^{4}$ K. Fissum, ${ }^{15}$ B. Fox, ${ }^{5}$ S. Frullani, ${ }^{12}$ H. Gao, ${ }^{18}$ J. Gao, ${ }^{3}$ F. Garibaldi, ${ }^{12}$ O. Gayou, ${ }^{2,29}$ R. Gilman, ${ }^{25,27}$ A. Glamazdin, ${ }^{14}$ C. Glashausser, ${ }^{25}$ J. Gomez, ${ }^{27}$ V. Gorbenko, ${ }^{14}$ O. Hansen, ${ }^{27}$ R. J. Holt, ${ }^{1,11}$ J. Hovdebo, ${ }^{24}$ G. M. Huber, ${ }^{24}$ C. W. de Jager, ${ }^{27}$ X. Jiang, ${ }^{25}$ C. Jones, ${ }^{3}$ M. K. Jones, ${ }^{22}$

J. Kelly, ${ }^{16}$ E. Kinney, ${ }^{5}$ E. Kooijman, ${ }^{13}$ G. Kumbartzki, ${ }^{25}$ M. Kuss, ${ }^{27}$ J. LeRose, ${ }^{27}$ M. Liang, ${ }^{27}$ R. Lindgren, ${ }^{28}$ N. Liyanage, ${ }^{27}$ S. Malov, ${ }^{25}$ D. J. Margaziotis, ${ }^{4}$ P. Markowitz, ${ }^{8}$ K. McCormick, ${ }^{6}$ D. Meekins, ${ }^{9}$ Z. -E. Meziani, ${ }^{26}$ R. Michaels, ${ }^{27}$

J. Mitchell, ${ }^{27}$ L. Morand, ${ }^{25}$ C. F. Perdrisat, ${ }^{29}$ R. Pomatsalyuk, ${ }^{14}$ V. Punjabi, ${ }^{20}$ R. D. Ransome, ${ }^{25}$ R. Roche, ${ }^{9}$ M. Rvachev, ${ }^{18}$ A. Saha, ${ }^{27}$ A. Sarty, ${ }^{9, \dagger}$ E. C. Schulte, ${ }^{1,11}$ D. Simon, ${ }^{10}$ S. Strauch, ${ }^{25, \$}$ R. Suleiman, ${ }^{13}$ L. Todor, ${ }^{22}$ P. E. Ulmer, ${ }^{22}$ G. M. Urciuoli, ${ }^{12}$ B. Wojtsekhowski, ${ }^{27}$ F. Xiong, ${ }^{18}$ and W. $\mathrm{Xu}^{18}$

(The Jefferson Lab Hall A Collaboration)

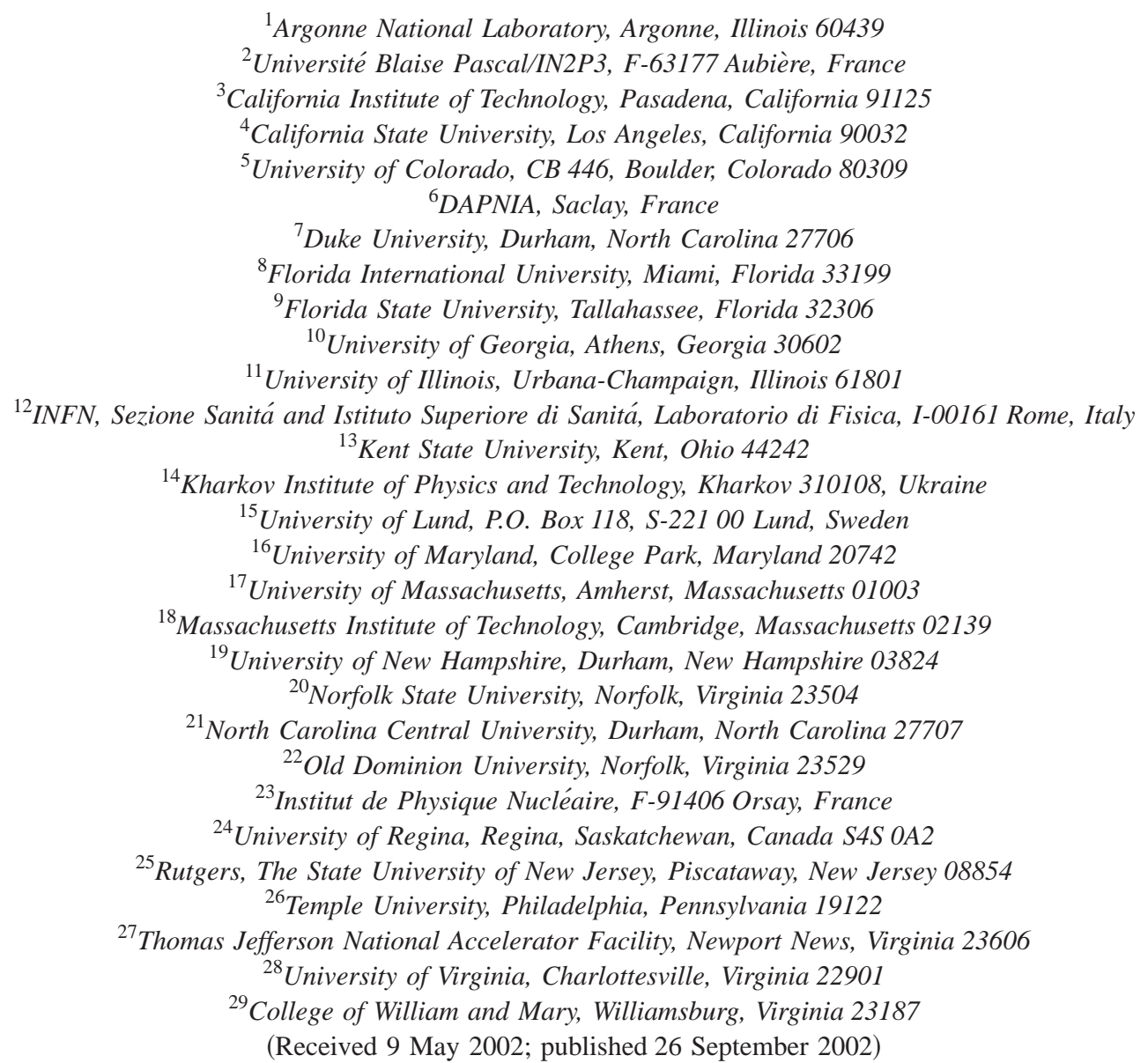

We present measurements of the recoil proton polarization for the ${ }^{1} H(\vec{\gamma}, \vec{p}) \pi^{0}$ reaction for $\theta_{\mathrm{c} . \mathrm{m} \text {. }}^{\pi}$ $=60^{\circ}-135^{\circ}$ and for photon energies up to $4.1 \mathrm{GeV}$. These are the first data in this reaction for polarization transfer with circularly polarized photons. Various theoretical models are compared with the results. No evidence for hadron helicity conservation is observed. Models that employ factorization are not favored. It appears from the strong angular dependence of the induced polarization at photon energies of 2.5 and $3.1 \mathrm{GeV}$ that a relatively high spin resonance or background amplitude might exist in this energy region.

DOI: $10.1103 /$ PhysRevC.66.034614

PACS number(s): 13.60.Le, 24.70.+s, 24.85.+p, 25.20.Lj

\footnotetext{
*Corresponding author. Email address: krishniw@jlab.org

†Present address: St. Mary's University, Halifax, Nova Scotia, Canada B3H 3C3.

₹Present address: George Washington University, Washington DC 20052.
} 
We present a survey of measurements of recoil proton polarization in neutral pion photo-production from the proton, the $\vec{\gamma} p \rightarrow \pi^{0} \vec{p}$ reaction. At lower photon energies, $E_{\gamma}$ $\leqslant 2 \mathrm{GeV}$, corresponding to $W=\sqrt{s}=2.15 \mathrm{GeV}, \pi^{0}$ photoproduction is dominated by the production and decay of baryon resonances, indicated by the structure in the cross section [1]. Further evidence for this can be found in polarization observables, which have however largely been measured only for photon energies below about $1.5 \mathrm{GeV}$ and for two observables, the induced recoil proton polarization $p_{y}$ and the linearly polarized photon asymmetry $\Sigma$.

Our data extend above the known resonance region, $W$ $>2 \mathrm{GeV}$, at large scattering angles and four momentum transfers. In this kinematic regime, the cross sections are known to approximately follow the constituent counting rules [2], which can be derived from perturbative QCD (pQCD). Scaling behavior of differential cross sections has been observed for a number of exclusive reactions at high transverse momenta [3-8].

Another simple consequence of $\mathrm{pQCD}$ is the prediction of hadron helicity conservation (HHC) [9]. HHC has been virtually untested in hadronic photoreactions until the recent advent of high intensity electron beams and polarimeters. HHC requires quark helicity conservation, and neglect of orbital angular momenta. It is generally accepted that HHC does not hold for hadron-hadron interactions [10]. Here, long-distance phenomena [11-13] are present, as opposed to the case where a single photon can interact only with a single quark in the target [14]. HHC predicts that the induced polarization $p_{y}$ and the transferred polarization $C_{x^{\prime} \text { c.m. }}$. vanish; the transferred polarization component $C_{z^{\prime} \text { c.m. }}$ is not constrained by HHC, but it should become nearly independent of beam energy. However, a very recent paper by Miller and Frank [15], using a Poincare invariant wave function suggests that helicity conservation is not satisfied for exclusive processes involving protons.

From Ref. [16], we can expect to have large polarizations for $C_{z^{\prime} \text { c.m. }}$, with the exact magnitude being a function of the hadronic distribution amplitudes. Another evaluation of $C_{z^{\prime} \text { c.m. }}$ comes by applying the formalism of Ref. [17] to exclusive photo-production of neutral pions and using factorization and pQCD arguments; this predicts $C_{z^{\prime} \text { c.m. }}=0.6$ at $\theta_{\text {c.m. }}=90^{\circ}$ in the scaling region. A high energy $\left(E_{\gamma}\right.$ $\geqslant 4 \mathrm{GeV}$ ) model for photo production is presented by Ref. [18]. At low-momentum transfer, by the use of Regge trajectory exchanges, this model is able to give a qualitative description of unpolarized and polarized data for both the charged and neutral pion photoproduction reactions. At highmomentum transfer, an extrapolation based on saturating Regge trajectories was used.

In this paper we present measurements of $p_{y}$, along with the polarization transfers $C_{x^{\prime}, z^{\prime}}$, from circularly polarized photons to recoil protons. These are the first such polarization transfer data in $\pi^{0}$ photoproduction; previously, these observables have only been measured in deuteron photodisintegration [19]. The few measured observables do not uniquely determine the four complex amplitudes of $\pi^{0}$ photoproduction. Thus, phase shift analysis requires theoretical

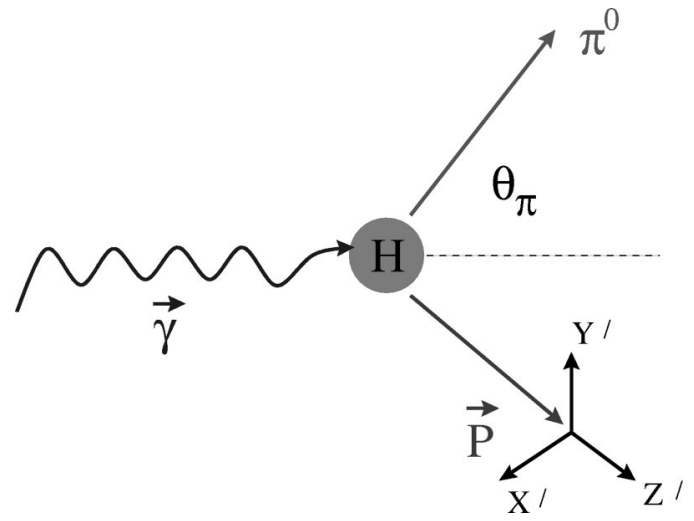

FIG. 1. Schematic diagram of the experiment, showing the coordinate system for the measured polarizations in the fixed lab frame.

assumptions. Our polarization transfer data are independent combinations of the reaction amplitudes. Thus, they provide a check of the theoretical assumptions. Above the known resonance region, if the quark models discussed are appropriate for understanding the reaction dynamics, one would expect the polarization observables to behave smoothly, possibly approaching limits imposed by hadronic helicity conservation. If instead the smooth cross sections result from an averaging of many underlying resonances, one might expect the polarization observables to have strong energy- and angle-dependent structures indicative of interference between resonant and background amplitudes.

\section{EXPERIMENTAL METHOD}

The measurements were performed in the experimental Hall A of the Thomas Jefferson National Accelerator Facility (JLab). A polarized electron beam source used a strained GaAs crystal to produce a polarized electron beam of $\approx 30 \mu \mathrm{A}$. The beam helicity state was flipped pseudorandomly at $30 \mathrm{~Hz}$. Charge asymmetries between the two helicity states were measured using two independent beam charge monitors in Hall A and were found to be negligible. The beam polarization $p_{e}$ was measured every few days with the Hall A M $\phi l l e r$ polarimeter, and averaged about $70 \%$, with typical uncertainties of $\pm 0.3 \%$ (statistics) and $\pm 3.0 \%$ (systematics).

Circularly polarized bremsstrahlung photons were generated when the electron beam impinged on a copper radiator with a thickness of $6 \%$ of a radiation length, positioned $\approx 73 \mathrm{~cm}$ upstream of a $15-\mathrm{cm}$ liquid hydrogen target. The ratio of the photon polarization $p_{\gamma}$ to the beam polarization can be directly calculated [20]; for the near-end-point photons of our experimental conditions, $p_{\gamma} / p_{e}$ is $98.4 \%-99.8 \%$.

Figure 1 shows the coordinate system in the laboratory frame for the experiment. (Note that all c.m. quantities will be subscripted c.m. while lab quantities will not be subscripted.) The convention for the axes for the polarization components is similar to that defined by Barker et al. [21], 


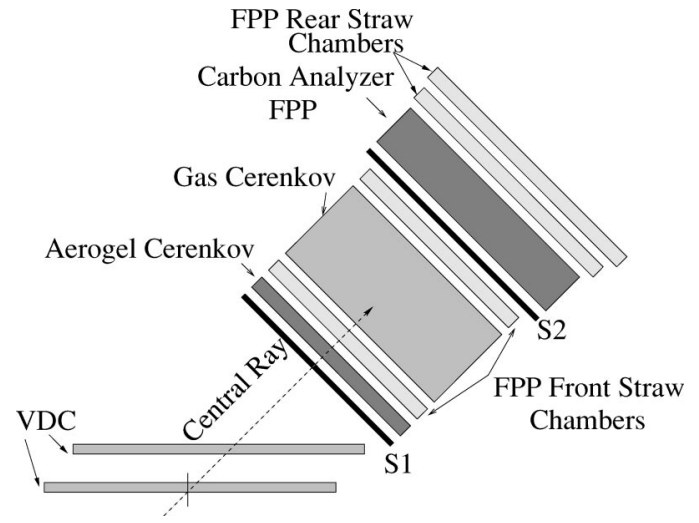

FIG. 2. Schematic figure showing the hadron arm detector package, polarimeter chambers, and the segmented analyzer.

which is however defined in the center-of-mass frame. The c.m. coordinate system is defined by $\hat{z}_{\text {c.m. }}=\hat{k}_{\gamma}, \hat{z}_{\text {c.m. }}^{\prime}=\hat{k}_{\pi^{0}}$, and $\hat{y}_{\mathrm{c} . \mathrm{m} .}=\hat{y}_{\mathrm{c} . \mathrm{m} .}^{\prime}=\hat{z}_{\mathrm{c} . \mathrm{m} .} \times \hat{z}_{\mathrm{c} . \mathrm{m} .}^{\prime}\left|\hat{z}_{\mathrm{c} . \mathrm{m} .} \times \hat{z}_{\mathrm{c} . \mathrm{m} .}^{\prime}\right|$. The $\hat{x}_{\mathrm{c} . \mathrm{m} .}=\hat{y}_{\mathrm{c} . \mathrm{m} .}$ $\times \hat{z}_{\text {c.m. }}$ and $\hat{x}_{\text {c.m. }}^{\prime}=\hat{y}_{\text {c.m. }}^{\prime} \times \hat{z}_{\text {c.m. }}^{\prime}$ vectors make the system right handed. If the beam helicity and the observables $C_{x^{\prime} \text { c.m. }}$, $p_{y \text { c.m. }}$, and $C_{z^{\prime} \text { c.m. }}$ are positive, the three proton polarization components then point in the $\hat{x}_{\mathrm{c} . \mathrm{m} .}^{\prime}, \hat{y}_{\mathrm{c} . \mathrm{m} \text {. }}$, and $-\hat{z}_{\mathrm{c} . \mathrm{m} \text {. }}^{\prime}$ directions, due to an explicit minus sign in the definition of $C_{z^{\prime} \text { c.m. }}$. Our lab conventions, shown in Fig. 1, include:

(1) $\hat{y}=\hat{y}_{\text {c.m. }}$, since there are no boost effects on this component;

(2) $\hat{x}^{\prime}$ positive to larger angles (Note that this convention is opposite to that used by phase shift analysis codes, SAID [22] and MAID [23].), so that in the low energy limit $\hat{x}_{\text {c.m. }}^{\prime} \rightarrow \hat{x}^{\prime}$; and

(3) $\hat{z}^{\prime}=\hat{k}_{\text {proton }}$, because the proton and $\pi^{0}$ are not collinear in the lab as in c.m., and so that positive $C_{z}^{\prime}$ means the polarization points in the $\hat{z}^{\prime}$ direction.

Note that this choice of axes results, however, in a lefthanded coordinate system.

Photoprotons emitted from the target were detected in the Hall A high resolution hadron spectrometer (HRSH). The trajectories were measured with Vertical Drift Chambers (VDCs) located in the focal plane of the spectrometer. The scattering angles, momentum, and interaction position at the target were calculated from the VDC tracks. Two planes of plastic scintillators provided triggering and time-of-flight information for particle identification. An aerogel Cerenkov counter was used to identify and reject pions, which constitute a maximum of $30 \%$ background in certain kinematic settings of these measurements.

The final element in the detector stack was the proton polarimeter (shown in Fig. 2), consisting of two front and two rear straw chambers that determined the scattering angles in a carbon analyzer. The analyzer consists of five sets of carbon plates. Each set is split at the middle into two plates, which can be moved in and out as desired. The thicknesses of the plates from the front to the rear are 22.9, 15.2, $7.6,3.8$, and $1.9 \mathrm{~cm}$.

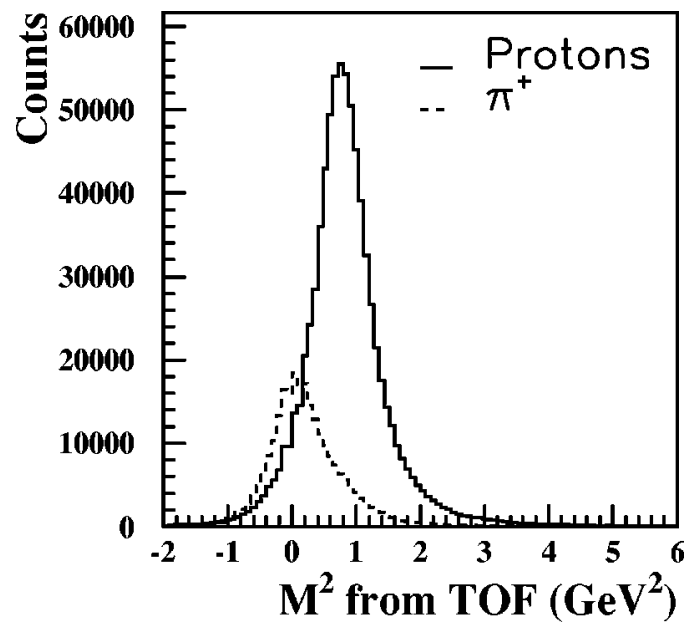

FIG. 3. Mass spectrum at $E_{\gamma}=2.5 \mathrm{GeV}$ as determined from time-of-flight measurement and momentum reconstruction in HRSH. The aerogel Cerenkov cut permits the separation of protons from positive pions.

Components of the proton polarization transverse to the momentum at the analyzer led to azimuthal asymmetries in the distribution of protons scattered from the carbon analyzer. An event trigger was formed by a coincidence of scintillators $S 1$ and $S 2$. The scintillators, as shown in Fig. 2, were located before the analyzer, to prevent possible false asymmetries, at the expense of reading in events in which the protons were absorbed in the analyzer. The efficiency was large because of the large rear chambers, which detected all protons scattered at angles less than $15^{\circ}$, and had high geometric acceptance for scatterings up to $30^{\circ}$ in the analyzer.

The major source of background is protons from $e p$ elastic scattering in the LH2 target. Some $e p$ data were collected with the radiator out, to measure the strength of the $e p$ radiative tail and to subtract the $e p$ radiative tail events from the $H(\gamma, p) \pi^{0}$ data. Another source of background arises from $\pi^{+}$,s from the $H\left(\gamma, \pi^{+}\right) n$ reaction. These $\pi^{+}$events were removed using the aerogel Cerenkov detector. The background particles coming from $\mathrm{Al}$ end caps of the target were measured using empty target runs.

\section{DATA ANALYSIS}

\section{A. Determination of kinematic variables and cuts}

Proton identification was obtained by reconstructing the mass from the time-of-flight measurement between the two pairs of scintillator planes in the spectrometer and from the reconstructed momentum of the particle. Figure 3 shows such a reconstructed mass spectrum at $E_{\gamma}=2.5 \mathrm{GeV}$.

The incident photon energy was reconstructed from the scattered proton energy and angle, using two-body pion photo-production kinematics. Only the events between the bremsstrahlung end point and the two-pion production threshold were used in the analysis. Since the elastic proton 
TABLE I. Kinematics and polarimeter parameters.

\begin{tabular}{|c|c|c|c|c|c|c|c|c|c|c|}
\hline $\begin{array}{c}\bar{E}_{\gamma} \\
(\mathrm{GeV})\end{array}$ & $\begin{array}{c}E_{\gamma} \text { range } \\
(\mathrm{GeV})\end{array}$ & $\begin{array}{c}\bar{W} \\
(\mathrm{GeV})\end{array}$ & $\begin{array}{c}p_{T} \\
(\mathrm{GeV} / c)\end{array}$ & $\begin{array}{c}-t_{\pi} \\
(\mathrm{GeV} / c)^{2}\end{array}$ & $\begin{array}{c}\theta_{\pi} \text { c.m. } \\
(\mathrm{deg})\end{array}$ & $\begin{array}{l}\theta_{p} \text { lab } \\
(\mathrm{deg})\end{array}$ & $\begin{array}{c}P_{p} \text { lab } \\
(\mathrm{GeV} / c)\end{array}$ & $\begin{array}{c}\text { Analyzer } \\
\text { thickness }(\mathrm{cm})\end{array}$ & $\bar{A}_{y}$ & $\begin{array}{c}\bar{\chi} \\
(\operatorname{deg})\end{array}$ \\
\hline 0.823 & $0.803-0.848$ & 1.557 & 0.344 & 0.855 & 135.0 & 19.9 & 1.012 & 26.7 & 0.37 & 116.6 \\
\hline 0.818 & $0.795-0.848$ & 1.554 & 0.423 & 0.751 & 120.1 & 26.8 & 0.938 & 15.2 & 0.40 & 114.4 \\
\hline 0.813 & $0.780-0.848$ & 1.551 & 0.469 & 0.631 & 105.4 & 33.6 & 0.847 & 11.4 & 0.46 & 109.5 \\
\hline 0.807 & $0.750-0.848$ & 1.547 & 0.482 & 0.501 & 91.3 & 40.4 & 0.744 & 7.6 & 0.52 & 103.8 \\
\hline 0.807 & $0.750-0.848$ & 1.547 & 0.475 & 0.372 & 76.2 & 48.4 & 0.635 & 3.8 & 0.49 & 97.4 \\
\hline 0.773 & $0.730-0.848$ & 1.526 & 0.448 & 0.365 & 74.9 & 48.5 & 0.598 & 3.8 & 0.43 & 95.3 \\
\hline 0.806 & $0.740-0.848$ & 1.547 & 0.427 & 0.251 & 61.1 & 56.1 & 0.514 & 3.8 & 0.25 & 91.6 \\
\hline 1.098 & $1.060-1.140$ & 1.715 & 0.595 & 0.746 & 90.1 & 39.9 & 0.928 & 22.9 & 0.42 & 112.8 \\
\hline 1.050 & $0.994-1.100$ & 1.688 & 0.506 & 0.370 & 60.7 & 55.3 & 0.616 & 7.6 & 0.41 & 95.9 \\
\hline 1.227 & $1.200-1.250$ & 1.784 & 0.619 & 1.071 & 105.1 & 32.2 & 1.161 & 26.7 & 0.29 & 127.3 \\
\hline 1.217 & $1.185-1.250$ & 1.778 & 0.637 & 0.848 & 89.9 & 39.5 & 1.001 & 22.9 & 0.37 & 116.2 \\
\hline 1.205 & $1.160-1.250$ & 1.772 & 0.612 & 0.628 & 75.0 & 47.1 & 0.835 & 11.4 & 0.46 & 106.6 \\
\hline 1.639 & $1.620-1.660$ & 1.989 & 0.537 & 2.059 & 135.1 & 17.6 & 1.777 & 49.5 & 0.17 & 170.8 \\
\hline 1.638 & $1.615-1.660$ & 1.988 & 0.662 & 1.810 & 119.9 & 24.0 & 1.628 & 49.5 & 0.19 & 160.0 \\
\hline 1.633 & $1.610-1.660$ & 1.986 & 0.739 & 1.519 & 104.9 & 30.6 & 1.451 & 49.5 & 0.25 & 147.8 \\
\hline 1.629 & $1.590-1.660$ & 1.984 & 0.756 & 1.205 & 90.6 & 37.4 & 1.244 & 34.3 & 0.26 & 129.0 \\
\hline 1.613 & $1.560-1.660$ & 1.976 & 0.734 & 0.893 & 74.8 & 45.4 & 1.031 & 22.9 & 0.34 & 117.0 \\
\hline 1.603 & $1.520-1.660$ & 1.972 & 0.654 & 0.601 & 59.6 & 53.7 & 0.811 & 11.4 & 0.48 & 104.3 \\
\hline 1.921 & $1.900-1.940$ & 2.117 & 0.598 & 2.490 & 134.9 & 17.1 & 2.033 & 49.5 & 0.15 & 189.8 \\
\hline 1.918 & $1.895-1.940$ & 2.116 & 0.732 & 2.189 & 119.9 & 23.2 & 1.858 & 49.5 & 0.19 & 176.9 \\
\hline 1.913 & $1.885-1.940$ & 2.114 & 0.817 & 1.838 & 104.8 & 29.7 & 1.649 & 49.5 & 0.19 & 162.2 \\
\hline 1.905 & $1.870-1.940$ & 2.110 & 0.842 & 1.459 & 89.7 & 36.7 & 1.409 & 41.9 & 0.24 & 144.2 \\
\hline 1.891 & $1.840-1.930$ & 2.104 & 0.812 & 1.081 & 74.8 & 44.3 & 1.162 & 34.3 & 0.29 & 126.6 \\
\hline 1.876 & $1.790-1.930$ & 2.097 & 0.722 & 0.728 & 59.6 & 52.6 & 0.909 & 15.2 & 0.41 & 110.4 \\
\hline 2.472 & $2.450-2.490$ & 2.349 & 0.691 & 3.347 & 134.9 & 15.9 & 2.522 & 49.5 & 0.10 & 227.8 \\
\hline 2.469 & $2.450-2.490$ & 2.348 & 0.848 & 2.941 & 119.9 & 21.7 & 2.293 & 49.5 & 0.12 & 210.0 \\
\hline 2.466 & $2.446-2.490$ & 2.346 & 0.950 & 2.468 & 104.7 & 28.0 & 2.023 & 49.5 & 0.15 & 190.0 \\
\hline 2.460 & $2.400-2.490$ & 2.344 & 0.980 & 1.959 & 89.6 & 34.8 & 1.717 & 49.5 & 0.18 & 166.0 \\
\hline 2.453 & $2.400-2.490$ & 2.341 & 0.944 & 1.451 & 74.6 & 42.2 & 1.405 & 41.9 & 0.24 & 143.2 \\
\hline 2.436 & $2.350-2.490$ & 2.334 & 0.842 & 0.977 & 59.5 & 50.5 & 1.091 & 26.7 & 0.32 & 122.3 \\
\hline 3.080 & $3.000-3.095$ & 2.580 & 1.081 & 3.168 & 104.7 & 26.4 & 2.432 & 49.5 & 0.12 & 221.7 \\
\hline 3.075 & $3.000-3.095$ & 2.578 & 1.121 & 2.517 & 89.2 & 33.1 & 2.052 & 49.5 & 0.15 & 193.1 \\
\hline 3.062 & $3.000-3.095$ & 2.574 & 1.084 & 1.867 & 74.0 & 40.6 & 1.665 & 49.5 & 0.19 & 164.9 \\
\hline 3.045 & $2.960-3.095$ & 2.567 & 0.962 & 1.261 & 59.2 & 48.7 & 1.281 & 41.9 & 0.26 & 135.6 \\
\hline 4.028 & $3.960-4.070$ & 2.905 & 1.129 & 1.728 & 59.1 & 45.9 & 1.572 & 49.5 & 0.22 & 158.8 \\
\hline
\end{tabular}

peak is higher in energy than the bremsstrahlung end-point protons from the $H(\gamma, p) \pi^{0}$ reaction, all $e p$ events, except for the ones in the radiative tail, are removed by this software cut. Other reactions such as heavier meson photoproduction are also removed by this software cut. Real Compton scattering (RCS) events are not removed, but since the RCS cross sections are small, these events are about a $1 \%$ background. The kinematics of the data points are given in Table I.

\section{B. Determination of the background}

For the subtraction of the radiative tail from the ep elastic peak we used a Monte Carlo simulation matched to data at the elastic peak (shown in Fig. 4). The polarizations for the ep elastic tail were assumed to be equal to the polarizations measured for the ep elastic peak. Afanasev et al. [24] have calculated the single photon emission corrections to the two polarization observables and shown that these corrections are of the order of $1 \%$. In this analysis, $\pi^{0}$ electro- and photoproduction polarizations were expected and measured to be equal and the data points reported are for the combined data set of electro- and photoproduction. The backgrounds from target cell walls were small, and were subtracted out.

\section{Asymmetries at the focal plane}

Polar and azimuthal angles for scattering in the $\mathrm{C}$ analyzer were measured by detecting the trajectory of the proton before and after the analyzer. Only the events within polar angle range of $5^{\circ}-20^{\circ}$ were used (see Fig. 5) for analysis. The analyzing power for this angle region of $\theta_{f p p}$ is well calibrated over a wide range of energies. The minimum is chosen so that the Coulomb scattering in the analyzer is re- 


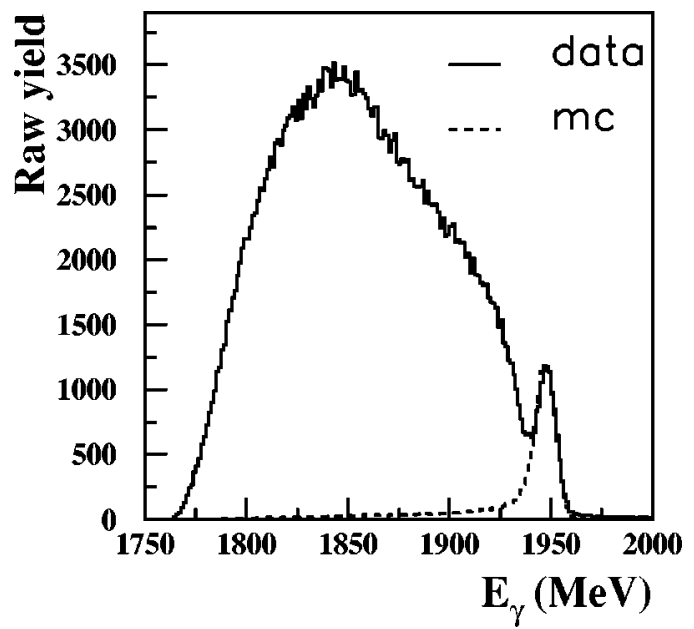

FIG. 4. Radiator-in bremsstralung spectrum at $E_{e}=1.95 \mathrm{GeV}$ at $\theta_{\text {c.m. }}=135^{\circ}$ data with the Monte Carlo simulation for the elastic events. Only the electron scattering part is shown for the simulation.

jected. The maximum is chosen to remove the large-angle region where the analyzing power and the efficiency are dropping rapidly.

The azimuthal angular distribution of the secondary scattering off the analyzer is given by

$$
\begin{aligned}
N_{p}\left(\theta_{f p p}, \phi_{f p p}\right) & \\
= & N_{p}\left(\theta_{f p p}\right)\left\{1+\left[A_{y}\left(\theta_{f p p}\right) P_{x}^{f p}+a_{i n s t}\right] \cos \phi_{f p p}\right. \\
& \left.-\left[A_{y}\left(\theta_{f p p}\right) P_{y}^{f p}+b_{i n s t}\right] \sin \phi_{f p p}\right\}
\end{aligned}
$$

where $N_{p}\left(\theta_{f p p}\right)$ is the number of protons scattered in the polarimeter, $A_{y}\left(\theta_{f p p}\right)$ is the analyzing power, and $a_{\text {inst }}, b_{\text {inst }}$ are the false asymmetries. The induced (transferred) polarization can be determined by the sum (difference) of the azimuthal asymmetry distributions for the two beam helicity states. Typical distributions are shown in Fig. 6. Note that Fig. 6 shows a clear sinusoidal distribution for the polariza-

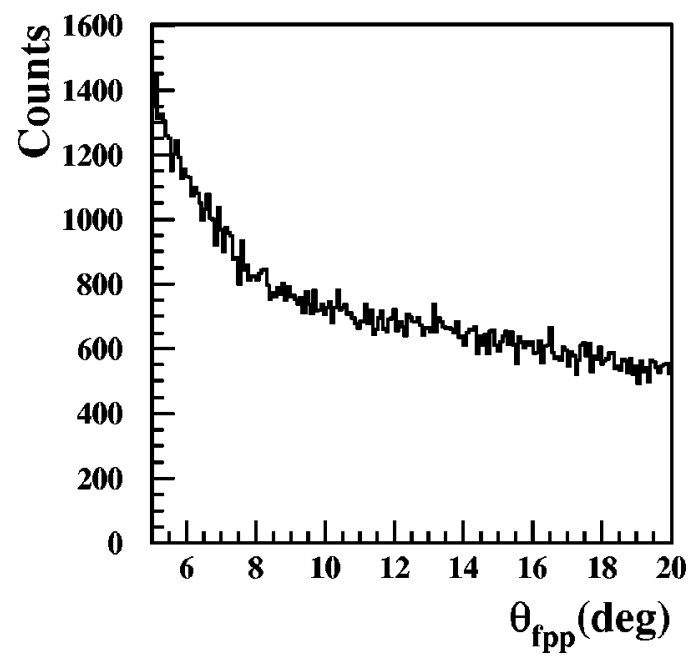

FIG. 5. Polarimeter polar scattering angle, $\theta_{f p p}$ for the $2.5 \mathrm{GeV}$, $\theta_{\text {c.m. }}=90^{\circ}$ data.

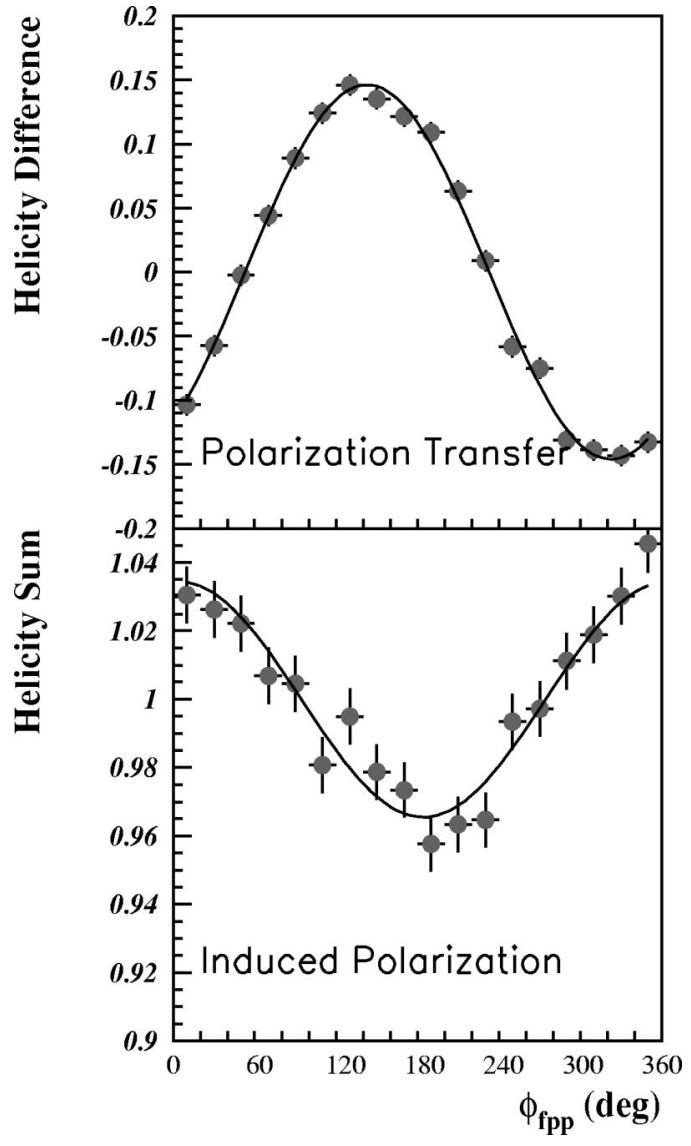

FIG. 6. Polarimeter azimuthal difference and sum distributions for positive and negative beam helicity, for the $0.86 \mathrm{GeV}, \theta_{\text {c.m. }}$. $=135^{\circ}$ data. The fits shown consist of a polynomial of $\sin (\phi)$ and $\cos (\phi)$ terms.

tion transfer components, but the induced polarization indicates other Fourier components, coming from the false asymmetries.

Determination of the proton polarization required a careful treatment of the polarimeter systematics. A description of the polarimeter systematics is also given in Ref. [19]. Carbon analyzing powers for the low-momentum points were obtained from the McNaughton parametrization [25]. For the

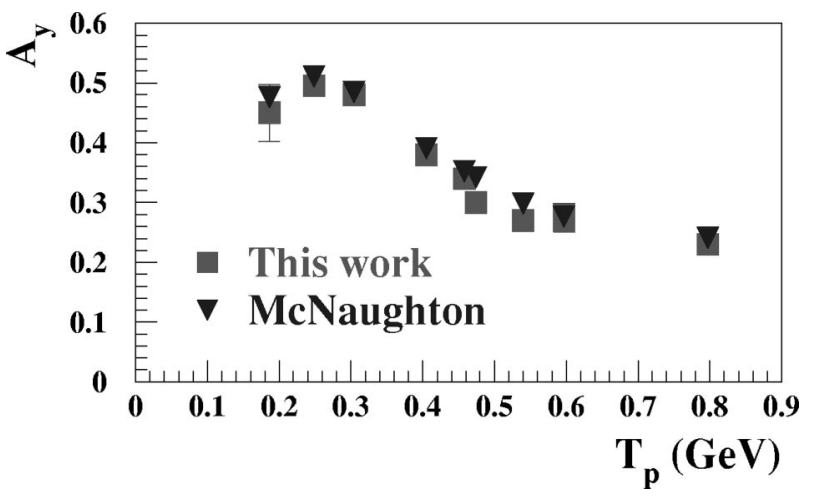

FIG. 7. Comparison of the analyzing power, averaged over $\theta_{f p p}=5^{\circ}-20^{\circ}$, for these data with the McNaughton parametrization, as a function of the proton kinetic energy $T_{p}$. 
higher-momentum points analyzing powers were interpolated from the ep elastic data from previous measurements using the same analyzer [26] with similar analyzer thicknesses. While our measurements $[27,28]$ agree well with previous data for the form factor ratio [26] and for the carbon analyzing power $[25,26,29]$, as shown in Fig. 7, our measurements do not cover all of our kinematics. The eventaveraged analyzing powers in each kinematic setting, for a $\theta_{f p p}$ range of $5^{\circ}-20^{\circ}$, is given in Table I.

False asymmetries are small, with magnitudes typically $<0.01$ and a smooth variation across the acceptance. The data for the induced polarization are corrected for the measured false asymmetries. For the polarization transfer data, the false asymmetries largely cancel with the helicity difference.

\section{Polarization observables at the target}

The proton spin precesses through different magnetic elements of the HRSH. In the simple dipole approximation, the precession angle $\chi$ is related to the bend angle, $\theta_{\text {bend }}$ by

$$
\chi=\frac{g-2}{2} \gamma \theta_{\text {bend }} \text {. }
$$

As a result, the polarization components at the target are different from the values measured at the FPP. They are related through a $3 \times 3$ spin transport matrix $(\mathbf{S})$ that depends on the trajectory's target quantities, $\theta, \phi, y$, and $p$, resulting in $\mathbf{S}$ to be unique for each event. The differential algebrabased code COSY [30] was used to determine the matrix elements $S_{i j}$.

A maximum likelihood method was used to obtain the induced and transferred polarizations at the target. We require that any event in our data set passes a cone test. The test ensures that the event would be accepted for any $\phi_{f p p}$ given its incident trajectory and $\theta_{f p p}$. This eliminates geometrical inefficiencies and allows us to use a simple likelihood function $L\left(P_{x}^{t g}, P_{z}^{t g}, P_{y}^{t g}\right)$, defined as

$$
\begin{aligned}
L\left(P_{x}^{t g}, P_{z}^{t g}, P_{y}^{t g}\right)= & \prod_{n=1}^{N_{p}}\left\{1+A_{y}\left(\theta_{f p p, n}\right)\left(S_{x y, n} P_{y}^{t g}+S_{x x, n} P_{x}^{t g}\right.\right. \\
& \left.+S_{x z, n} P_{z}^{t g}+a_{i n s t}\right) \cos \phi_{f p p, n}-A_{y}\left(\theta_{f p p, n}\right) \\
& \times\left(S_{y y, n} P_{y}^{t g}+S_{y x, n} P_{x}^{t g}+S_{y z, n} P_{z}^{t g}\right. \\
& \left.\left.+b_{i n s t}\right) \sin \phi_{f p p, n}\right\},
\end{aligned}
$$

where the product runs over all events, $N_{p}$. Here, $a_{\text {inst }}$ and $b_{\text {inst }}$ are the false asymmetries for the polarimeter, $P^{t g}$ are the polarizations at the target, and $S_{i j}$ are the elements of the spin transport matrix.

The proton polarization at the target is given by

$$
P_{k}^{t g}=h C_{k}^{\text {transfer }}+P_{k}^{\text {induced }},
$$

where $P_{k}^{t g}$ is the total target polarization in the $\hat{k}$ direction and $C_{k}^{\text {transfer }}\left(P_{k}^{\text {induced }}\right)$ is the polarization transfer (induced polarization) component in the $\hat{k}$ direction.

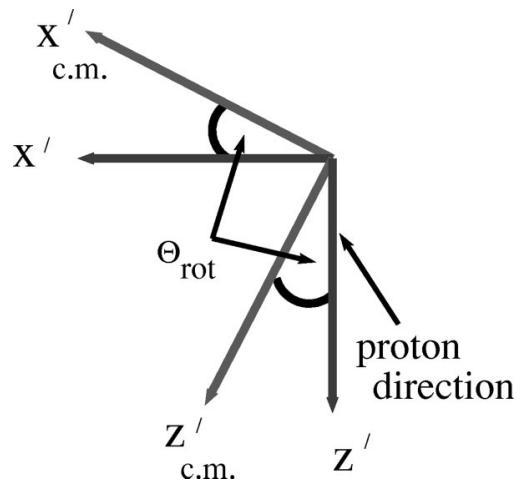

FIG. 8. Schematic showing the rotation angle in the transformation from c.m. frame to lab frame.

This procedure allows one to obtain both the induced and transferred polarizations at the target. The stability of the method was studied in detail for all kinematic points. Variation of the four target quantities mentioned above, by their systematic uncertainties, leads to only minor changes in the extracted polarizations, less than the statistical uncertainties of the data.

Analysis of the sensitivity to ep background subtraction, to different false-asymmetry models, alignment or tracking procedures, cuts, spin transport, and uncertainties in the carbon analyzing power, leads to an estimated systematic uncertainty of about \pm 0.046 for the induced polarizations $p_{y}$. The largest contributions are \pm 0.03 each from the ep background subtraction systematics and false-asymmetry systematics. The polarization transfer observables $C_{x^{\prime}}$ and $C_{z^{\prime}}$ have systematic uncertainties of 0.036 and 0.048 , respectively. Again the largest contribution arises from the ep background subtraction, leading to uncertainties of 0.03 and 0.04 , respectively.

\section{E. Transformation from the c.m. frame to the lab frame}

The polarimeter measures the transverse components of the proton's spin in the laboratory, but calculations are generally in the c.m. frame. Thus, it is necessary to transform our lab results to the c.m. frame, or the calculations to the lab frame. In this work, we will transform the calculations, for reasons we now explain. The conversion from c.m. to lab frame can be performed as a series of boosts and rotations of the proton's spin four-vector. The result of this transformation is a mixing of the $\hat{x}^{\prime}$ and $\hat{z}^{\prime}$ components of the proton's spin. Figure 8 schematically shows this rotation; calculated rotation angles are given below in Table II. While the spin transfer observables are affected, the induced polarization, in the $\hat{y}^{\prime}$ direction, and the zeroth component of the spin are unchanged. In some of our kinematics we have precise values for $C_{x^{\prime}}$ in the lab frame, but $C_{z^{\prime}}$ is undetermined due to unfavorable spin transport. Mixing these components to compare to theory in the c.m. frame can give two components each of which have large uncertainties. Thus, the lab frame observables best constrain the theory, and we have decided to convert the theories into the lab frame. We report 
TABLE II. Polarization data for neutral pion photoproduction from this work. For the five $C_{x^{\prime}}$ points in

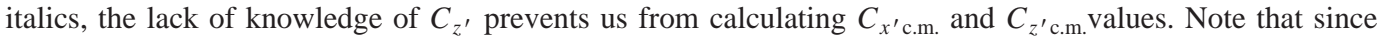
our $\hat{x}^{\prime}$ is directed opposite to that of SAID and MAID, our $C_{x^{\prime}}$ values are related to theirs by a factor of -1 .

\begin{tabular}{|c|c|c|c|c|c|c|c|}
\hline $\begin{array}{l}E_{\gamma} \\
(\mathrm{GeV})\end{array}$ & $\begin{array}{c}\theta_{\pi} \text { c.m. } \\
\quad(\mathrm{deg})\end{array}$ & $p_{y}$ & $C_{x^{\prime}}$ & $C_{z^{\prime}}$ & $C_{x^{\prime} \text { c.m. }}$ & $C_{z^{\prime} \text { c.m. }}$ & $\begin{array}{r}\theta_{r o t} \\
(\mathrm{deg})\end{array}$ \\
\hline 0.823 & 135.0 & $-0.246 \pm 0.049$ & $0.349 \pm 0.031$ & $0.428 \pm 0.037$ & $0.178 \pm 0.032$ & $0.523 \pm 0.036$ & 20.4 \\
\hline 0.818 & 120.1 & $-0.250 \pm 0.031$ & $0.331 \pm 0.018$ & $0.159 \pm 0.021$ & $0.220 \pm 0.019$ & $0.294 \pm 0.020$ & 27.5 \\
\hline 0.813 & 105.4 & $-0.435 \pm 0.046$ & $0.125 \pm 0.023$ & $0.017 \pm 0.025$ & $0.093 \pm 0.024$ & $0.085 \pm 0.024$ & 34.6 \\
\hline 0.807 & 91.3 & $-0.198 \pm 0.044$ & $-0.039 \pm 0.018$ & $0.076 \pm 0.018$ & $-0.080 \pm 0.018$ & $0.031 \pm 0.018$ & 41.7 \\
\hline 0.807 & 76.2 & $-0.337 \pm 0.110$ & $-0.002 \pm 0.032$ & $0.117 \pm 0.033$ & $-0.091 \pm 0.033$ & $0.074 \pm 0.032$ & 50.0 \\
\hline 0.773 & 74.9 & $-0.142 \pm 0.142$ & $0.038 \pm 0.027$ & $0.146 \pm 0.027$ & $-0.088 \pm 0.027$ & $0.122 \pm 0.027$ & 50.4 \\
\hline 0.806 & 61.1 & & $0.078 \pm 0.051$ & $0.066 \pm 0.050$ & $-0.014 \pm 0.050$ & $0.101 \pm 0.051$ & 57.6 \\
\hline 1.098 & 90.1 & $-0.399 \pm 0.024$ & & & & & 40.5 \\
\hline 1.050 & 60.7 & $0.012 \pm 0.088$ & & & & & 56.2 \\
\hline 1.227 & 105.1 & $0.305 \pm 0.027$ & $-0.503 \pm 0.027$ & $-0.144 \pm 0.034$ & $-0.346 \pm 0.029$ & $-0.392 \pm 0.032$ & 32.6 \\
\hline 1.217 & 89.9 & $-0.332 \pm 0.031$ & $-0.391 \pm 0.023$ & $0.492 \pm 0.027$ & $-0.616 \pm 0.025$ & $0.126 \pm 0.025$ & 40.0 \\
\hline 1.205 & 75.0 & $-0.261 \pm 0.049$ & $0.097 \pm 0.027$ & $0.400 \pm 0.028$ & $-0.230 \pm 0.028$ & $0.341 \pm 0.027$ & 47.7 \\
\hline 1.639 & 135.1 & $-0.489 \pm 0.023$ & $-0.107 \pm 0.029$ & $-0.096 \pm 0.151$ & $-0.073 \pm 0.054$ & $-0.124 \pm 0.144$ & 17.8 \\
\hline 1.638 & 119.9 & $0.311 \pm 0.022$ & $-0.248 \pm 0.027$ & $-0.222 \pm 0.081$ & $-0.135 \pm 0.041$ & $-0.304 \pm 0.075$ & 24.2 \\
\hline 1.633 & 104.9 & $0.396 \pm 0.017$ & $-0.644 \pm 0.019$ & $0.130 \pm 0.037$ & $-0.619 \pm 0.025$ & $-0.219 \pm 0.033$ & 30.9 \\
\hline 1.629 & 90.6 & $-0.295 \pm 0.091$ & $-0.686 \pm 0.079$ & $0.477 \pm 0.103$ & $-0.834 \pm 0.089$ & $-0.042 \pm 0.095$ & 37.7 \\
\hline 1.613 & 74.8 & $-0.492 \pm 0.040$ & $-0.196 \pm 0.027$ & $0.359 \pm 0.031$ & $-0.394 \pm 0.029$ & $0.110 \pm 0.029$ & 45.7 \\
\hline 1.603 & 59.6 & $0.017 \pm 0.068$ & $0.164 \pm 0.029$ & $0.031 \pm 0.030$ & $0.071 \pm 0.030$ & $0.151 \pm 0.029$ & 54.0 \\
\hline 1.921 & 134.9 & $-0.484 \pm 0.023$ & $-0.200 \pm 0.032$ & $-0.049 \pm 0.138$ & $-0.177 \pm 0.051$ & $-0.106 \pm 0.132$ & 17.2 \\
\hline 1.918 & 119.9 & $0.323 \pm 0.025$ & $-0.033 \pm 0.034$ & & & & 23.3 \\
\hline 1.913 & 104.8 & $0.544 \pm 0.021$ & $-0.700 \pm 0.027$ & $0.269 \pm 0.086$ & $-0.741 \pm 0.049$ & $-0.116 \pm 0.076$ & 29.9 \\
\hline 1.905 & 89.7 & $-0.196 \pm 0.018$ & $-0.615 \pm 0.021$ & $0.207 \pm 0.036$ & $-0.616 \pm 0.027$ & $-0.204 \pm 0.031$ & 36.9 \\
\hline 1.891 & 74.8 & $-0.473 \pm 0.036$ & $-0.271 \pm 0.032$ & $0.257 \pm 0.041$ & $-0.373 \pm 0.037$ & $-0.006 \pm 0.037$ & 44.5 \\
\hline 1.876 & 59.6 & $-0.186 \pm 0.049$ & $-0.173 \pm 0.029$ & $-0.084 \pm 0.031$ & $-0.038 \pm 0.030$ & $-0.189 \pm 0.030$ & 52.8 \\
\hline 2.472 & 134.9 & $1.036 \pm 0.092$ & $-0.020 \pm 0.102$ & $0.396 \pm 0.134$ & $-0.129 \pm 0.105$ & $0.375 \pm 0.132$ & 16.0 \\
\hline 2.469 & 119.9 & $0.040 \pm 0.056$ & $0.138 \pm 0.081$ & $0.673 \pm 0.149$ & $-0.122 \pm 0.093$ & $0.676 \pm 0.142$ & 21.8 \\
\hline 2.466 & 104.7 & $0.858 \pm 0.046$ & $-0.233 \pm 0.074$ & & & & 28.1 \\
\hline 2.460 & 89.6 & $-0.104 \pm 0.039$ & $-0.331 \pm 0.064$ & & & & 34.9 \\
\hline 2.453 & 74.6 & $0.193 \pm 0.048$ & $-0.177 \pm 0.065$ & $0.017 \pm 0.114$ & $-0.142 \pm 0.091$ & $-0.107 \pm 0.095$ & 42.4 \\
\hline 2.436 & 59.5 & $-0.143 \pm 0.032$ & $-0.214 \pm 0.030$ & $0.094 \pm 0.037$ & $-0.208 \pm 0.034$ & $-0.106 \pm 0.033$ & 50.7 \\
\hline 3.080 & 104.7 & $0.460 \pm 0.081$ & $-0.036 \pm 0.087$ & $0.394 \pm 0.127$ & $-0.208 \pm 0.096$ & $0.337 \pm 0.120$ & 26.4 \\
\hline 3.075 & 89.2 & $0.015 \pm 0.068$ & $-0.258 \pm 0.097$ & & & & 33.2 \\
\hline 3.062 & 74.0 & $0.279 \pm 0.031$ & $-0.212 \pm 0.043$ & $-0.044 \pm 0.150$ & $-0.132 \pm 0.103$ & $-0.171 \pm 0.117$ & 40.6 \\
\hline 3.045 & 59.2 & $-0.240 \pm 0.034$ & $-0.449 \pm 0.036$ & $0.023 \pm 0.053$ & $-0.313 \pm 0.046$ & $-0.323 \pm 0.044$ & 48.8 \\
\hline 4.028 & 59.1 & $0.040 \pm 0.114$ & $-0.661 \pm 0.134$ & & & & 46.0 \\
\hline
\end{tabular}

both lab and c.m. spin transfer components in Table II. Our frame transformation procedure is described in more detail in the Appendix.

\section{RESULTS AND DISCUSSION}

Existing theoretical predictions for the $H(\vec{\gamma}, \vec{p}) \pi^{0}$ reaction include: SAID [22], MAID [23], a quark model calculation by Afanasev, Carlson, and Wahlquist [17], and a pQCD prediction from Farrar, Huleihel, and Zhang [16]. A first attempt at including nuclear resonance effects on double polarizations has been performed by Dutta, Gao, and Lee [31].

In SAID, both an energy-dependent and a set of single energy partial wave analyses of single-pion photoproduction data are performed. These analyses extend from threshold to $2.0 \mathrm{GeV}$ in laboratory photon energy. Photo-decay amplitudes are extracted from Breit-Wigner fits for the baryon resonances within this energy range. For the neutral pion photo-production analysis, cross section $(d \sigma / d \Omega)$, photon asymmetry $(\Sigma)$, target asymmetry $(T)$, induced polarization $\left(p_{y}\right)$, and linear polarization transfer observables $\left(O_{x^{\prime}}\right.$, and $O_{z^{\prime}}$ ) data are used. Fits to existing data are used for this energy regime, and the resonance mass and width values were obtained from fits to a multipole analysis. Jenkins and Strakovsky [32] discussed the possible approach of SAID fits to helicity conservation at high energies. 


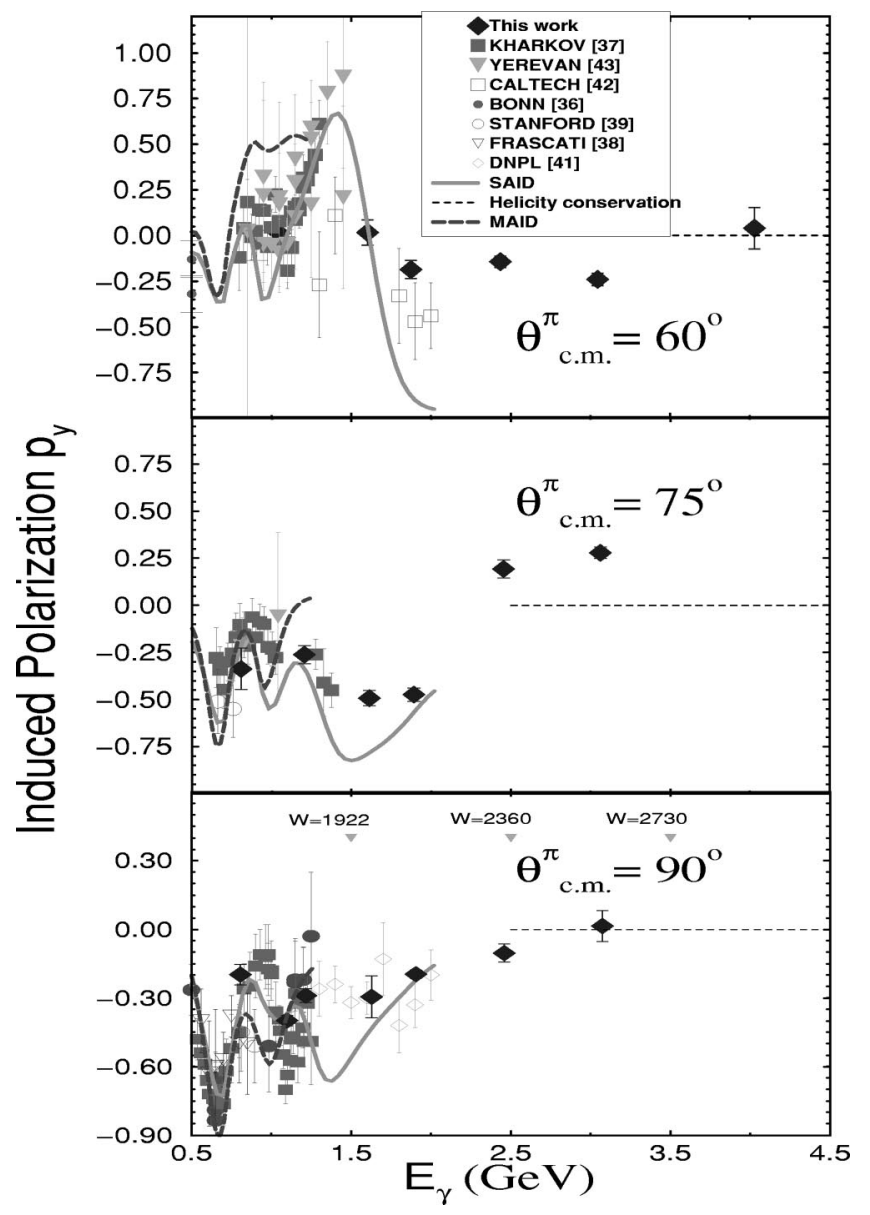

FIG. 9. Top to bottom: Induced polarization $p_{y}$ in neutral pion photo-production at $\theta_{\text {c.m. }}=60^{\circ}, 75^{\circ}$, and $90^{\circ}$. Only statistical uncertainties are shown. The three curves, SAID [22], MAID [23], and helicity conservation shown in the figures are described in the text. Corresponding $W$ range is also shown in the bottom plot.

The MAID model contains Born terms, vector mesons, and nucleon resonances up to the third resonance region $\left[P_{33}(1232), P_{11}(1440), D_{13}(1520), S_{11}(1535), F_{15}(1680)\right.$, and $\left.D_{33}(1700)\right]$. This model is expected to be valid up to $1.25 \mathrm{GeV}$ in photon energy. The resonance contributions are included taking into account unitarity to provide the correct phases of the pion photoproduction multipoles.

Afanasev et al. (see also Huang and Kroll [33]) use a pQCD approach for large transverse momenta $p_{T}$, where mesons are directly produced by short range processes. This approach is similar to the factorization approach [33] used to describe Compton scattering from the proton. The calculation assumes helicity conservation, which leads to the vanishing of $p_{y}$ and $C_{x^{\prime} \text { c.m. }}$. In the lab, $C_{x^{\prime}}$ does not generally vanish as it has contributions from both $C_{x^{\prime} \text { c.m. }}$ and $C_{z^{\prime} \text { c.m. }}$. This gives a simple result for exclusive photoproduction of neutral pions,

$$
\begin{gathered}
p_{y}=C_{x^{\prime} \text { c.m. }}=0, \\
C_{z^{\prime} \text { c.m. }}=\frac{s^{2}-u^{2}}{s^{2}+u^{2}} \lim _{x \rightarrow 1} \frac{e_{u}^{2} \Delta u_{v}(x)+e_{d}^{2} \Delta d_{v}(x)}{e_{u}^{2} u_{v}(x)+e_{d}^{2} d_{v}(x)},
\end{gathered}
$$

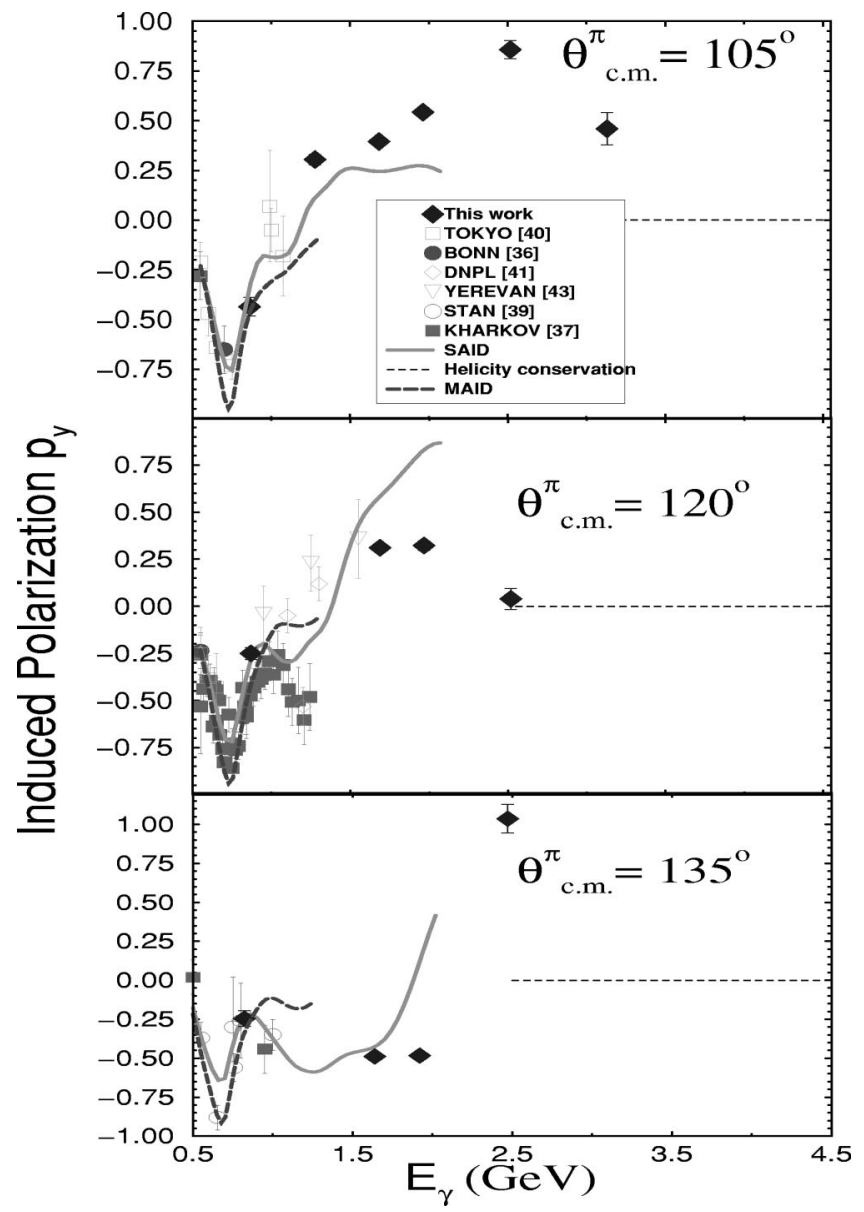

FIG. 10. Top to bottom: Induced polarization $p_{y}$ in neutral pion photo-production at $\theta_{\text {c.m. }}=105^{\circ}, 120^{\circ}, 135^{\circ}$. Only statistical uncertainties are shown. The three curves SAID [22], MAID [23], and helicity conservation shown here are described in the text.

where $e_{q}$ are the quark charges, $\Delta q(x)$ are the polarized distribution functions and $q(x)$ are the unpolarized distribution fuctions. When combined with pQCD constraints

$$
\lim _{x \rightarrow 1} \frac{\Delta q}{q} \rightarrow 1
$$

this simplifies to

$$
C_{z^{\prime} \text { c.m. }}=\frac{s^{2}-u^{2}}{s^{2}+u^{2}}
$$

This analysis predicts a value of $60 \%$ longitudinal polarization, $C_{z^{\prime} \text { c.m. }}$, for the recoil proton in the scaling region at $\theta_{\text {c.m. }}=90^{\circ}$. This model assumes that the polarization of the struck quark is the same as the polarization of the outgoing proton; however, wave function effects can dilute this effect.

Farrar et al. uses pQCD scaling arguments: $p_{y}$ and $C_{x^{\prime} \text { c.m. }}$. are zero at high photon energies and $C_{z^{\prime} \mathrm{c} . \mathrm{m} \text {. is constant at }}$ fixed $\theta_{\text {c.m. }}$. They use a dynamical model with explicit calculation of all lowest-order $\left(\alpha_{s}^{3}\right)$ Feynman diagrams. A general conclusion is that one may expect large polarization transfer in the pion photoproduction, with the exact magnitude being 


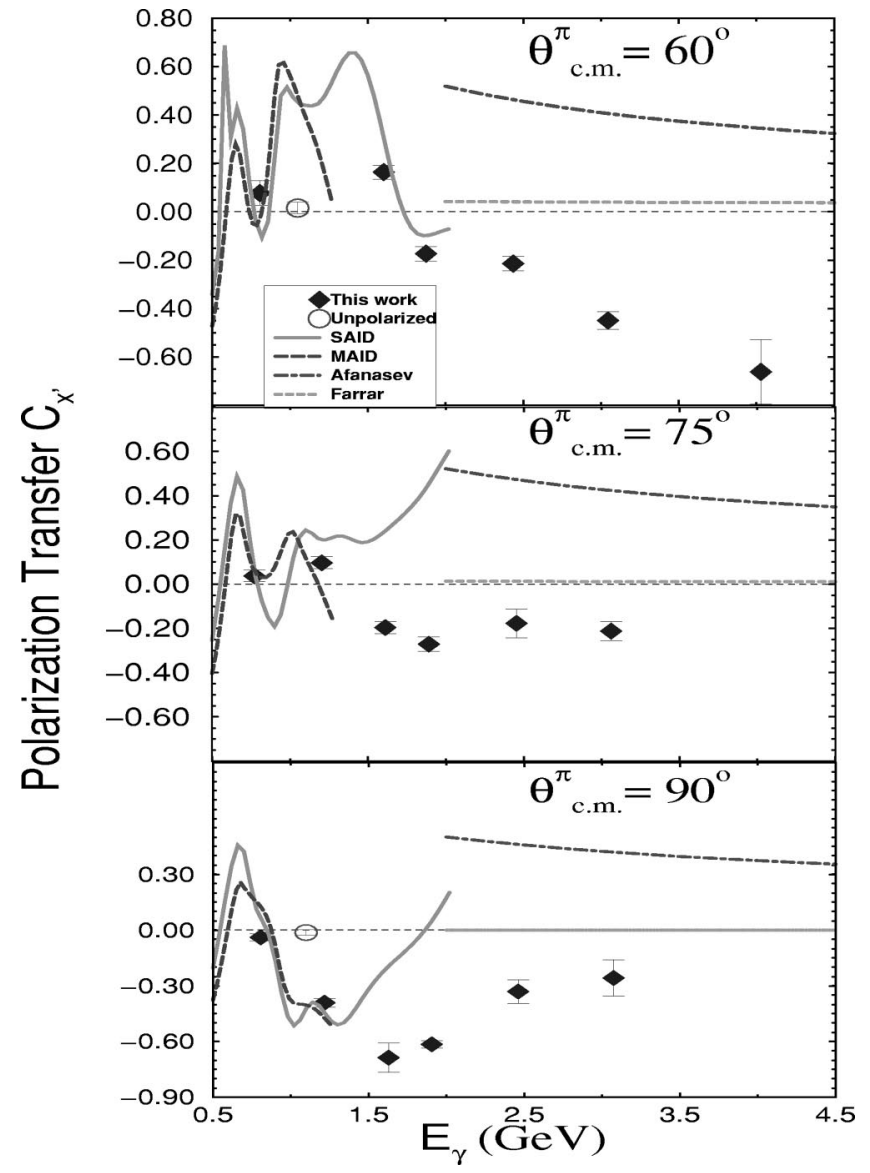

FIG. 11. Top to bottom: Polarization transfer $C_{x^{\prime}}$ in neutral pion photo-production at $\theta_{\text {c.m. }}=60^{\circ}, 75^{\circ}, 90^{\circ}$. Only statistical uncertainties are shown. The four curves SAID [22], MAID [23], Afanasev [17], and Farrar [16] are described in the text. All data and calculations are in the lab frame defined in Sec. I.

a function of hadronic distribution amplitudes. The calculations shown here used the asymptotic distribution amplitudes for both the proton and the pion. These pQCD approaches predict much smaller cross sections than the data and they are not expected to work until $-t$ of several $\mathrm{GeV}^{2}$.

Dutta [31] use the resonance parameters predicted by Capstick and Roberts [34,35]. Their analysis suggests that data for double polarizations on pion photo-production in the region $1.6 \leqslant W \leqslant 2.4 \mathrm{GeV}$ can be more useful in searching for missing resonances than the cross section, due to the large interference between the resonant and nonresonant background amplitudes. They connect resonant amplitudes directly to the predictions from the constituent quark model. This model assumes that the total amplitudes can be calculated from the multipole amplitudes generated by the SAID program. It also assumes that the energy dependence of the total decay width of most $N^{*}$ 's is similar to the width of the $N^{*} \rightarrow \pi N$ decay within the oscillator quark model. A cutoff parameter $\Lambda$ of $650 \mathrm{MeV}$ for all resonances and an average decay width of $300 \mathrm{MeV}$ for the known $N^{*}$ resonances and of $120 \mathrm{MeV}$ for the considered $\Delta^{*}$ resonances are also assumed. To date predictions are not available from this approach.

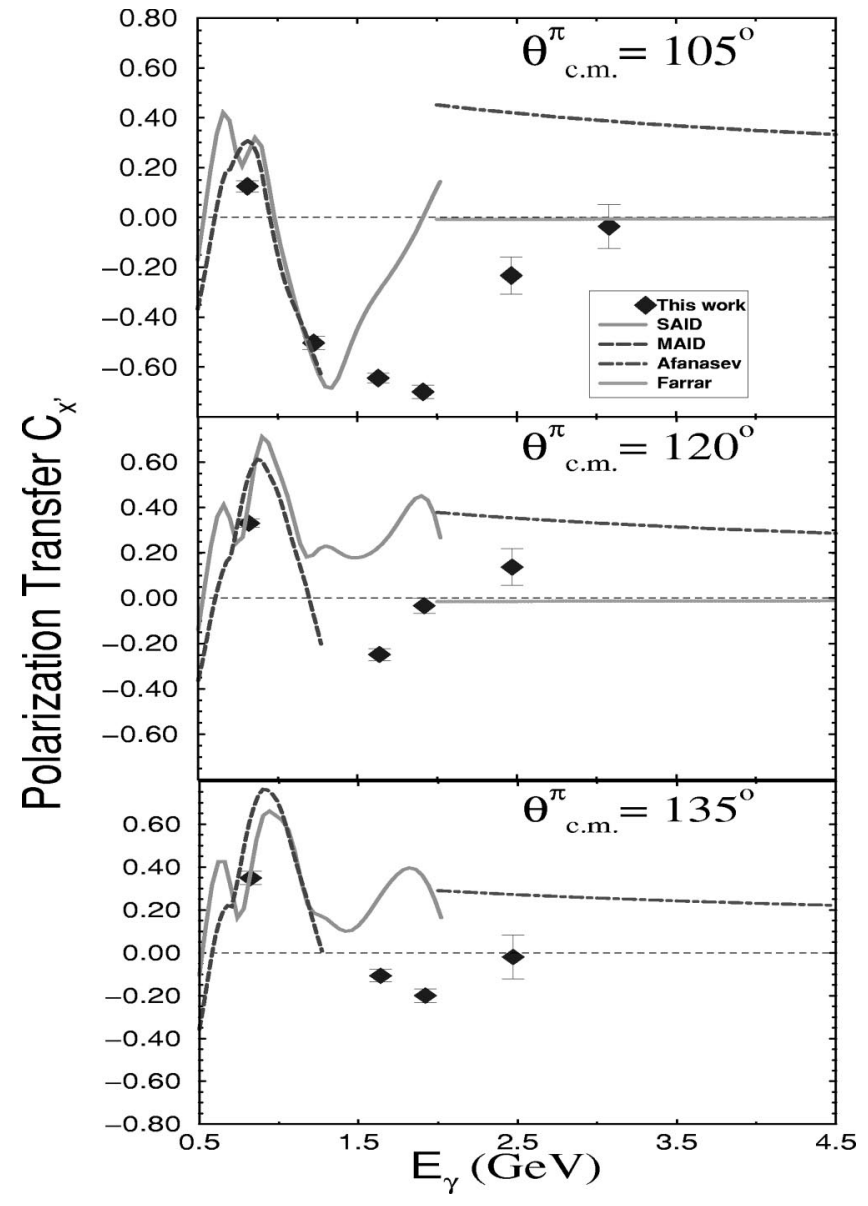

FIG. 12. Top to bottom: Polarization transfer $C_{x^{\prime}}$ in neutral pion photo-production at $\theta_{\text {c.m. }}=105^{\circ}, 120^{\circ}, 135^{\circ}$. Only statistical uncertainties are shown. The four curves SAID [22], MAID [23], Afanasev [17], and Farrar [16] are described in the text. All data and calculations are in the lab frame defined in Sec. I.

The polarization observables, both in the lab frame as well as in the c.m. frame, are given in Table II.

Figures 9 and 10 compare our data for the normal component of the induced polarization $p_{y}$ at different pion center-of-mass angles to previous measurements (BONN [36], KHARKOV [37], FRASCATI [38], STANFORD [39], TOKYO [40], DNPL [41], CALTECH [42], YEREVAN [43]) and theoretical predictions [22,23]. While our lowenergy data agree well with the world data, the highestenergy points seem to follow the trend predicted by the phase shift analysis code SAID, but not MAID (recall that MAID has been fitted only to $1.25 \mathrm{GeV}$ in photon energy, while SAID has data fitted up to $2.0 \mathrm{GeV}$ in photon energy). There is no general indication of an approach to helicity conservation, $p_{y} \rightarrow 0$. While the highest $-t$ achieved in this work is $-t$ $=3.4(\mathrm{GeV} / \mathrm{c})^{2}$ at $E_{\gamma}$ of $2.5 \mathrm{GeV}$ and at $\theta_{\mathrm{c} . \mathrm{m} .}^{\pi}=135^{\circ}$, the highest $p_{T}$ achieved is $p_{T}=1.1(\mathrm{GeV} / c)$ at $E_{\gamma}$ of $3.1 \mathrm{GeV}$ and at $\theta_{\mathrm{c} . \mathrm{m} .}^{\pi}=90^{\circ}$. This value of $p_{T}$ is somewhat lower than the scaling threshold of $p_{T}=1.3 \mathrm{GeV} / c$ observed [8] for the $\gamma d \rightarrow p n$ reaction. It is interesting that $p_{y}$ is zero at $90^{\circ}$, at approximately the same value of $p_{T}(\sim 1.1 \mathrm{GeV} / c)$ that $p_{y}$ for the $\gamma d \rightarrow p n$ reaction vanishes [19]. But, we cannot conclude from $p_{y}$ alone whether the pQCD limit has been observed. 


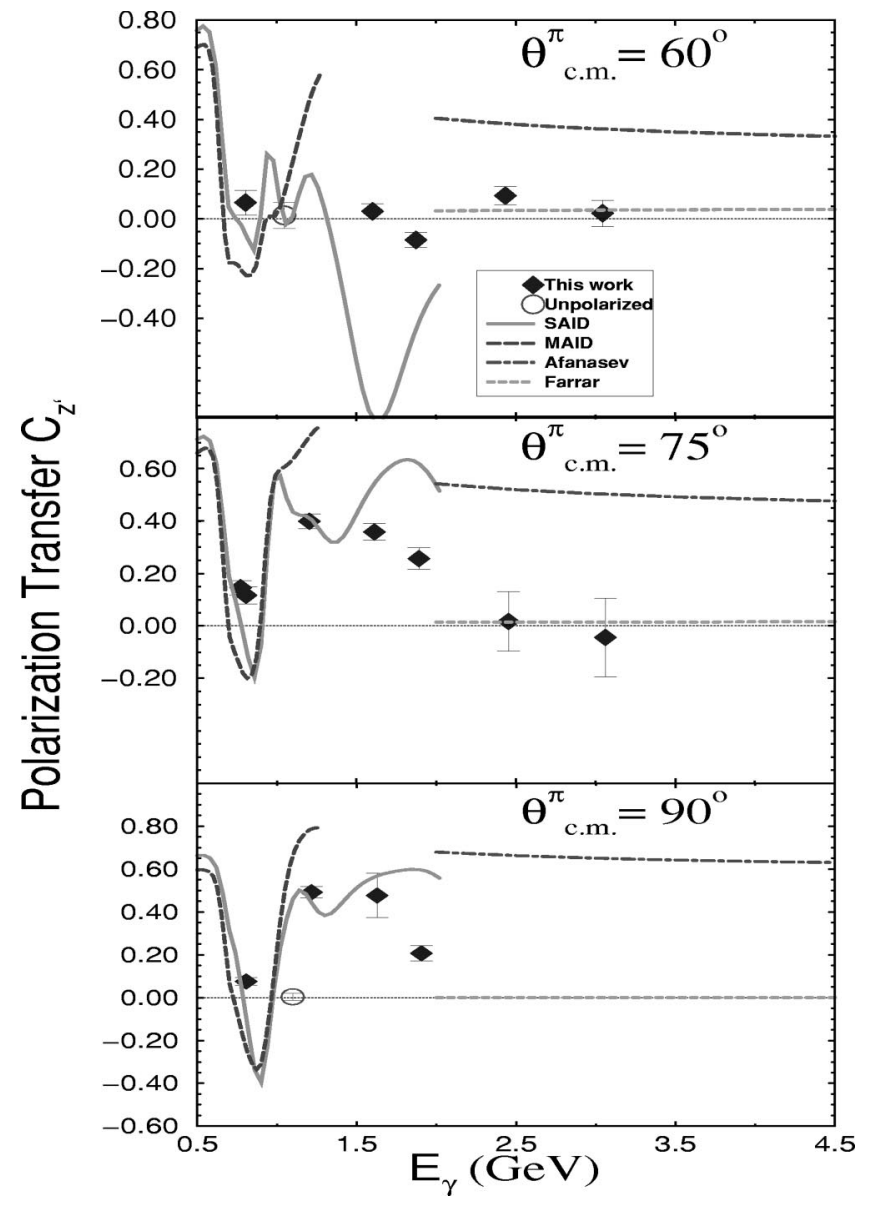

FIG. 13. Top to bottom: Polarization transfer $C_{z^{\prime}}$ in neutral pion photoproduction at $\theta_{\text {c.m. }}=60^{\circ}, 75^{\circ}, 90^{\circ}$. Only statistical uncertainties are shown. The four curves SAID [22], MAID [23], Afanasev [17], and Farrar [16] are described in the text. All data and calculations are in the lab frame defined in Sec. I.

The transverse in-plane polarization transfer, $C_{x^{\prime} \text { c.m. }}$, data shown in Table II also show no clear trend toward being small, positive, and only weakly dependent on energy and angle, as would be expected from the two helicityconserving quark-model calculations shown. This confirms the idea that a photon energy of $3.1 \mathrm{GeV}$ or a $p_{T}$ of $1.1 \mathrm{GeV} / c$ is still not sufficient for this reaction to exhibit HHC. Figures 11 and 12 show that $C_{x^{\prime}}$ data not only tend to agree reasonably with the SAID and MAID analyses at our lowest beam energies, but also tend to increasingly diverge from these analyses as the energy increases. Thus the amplitudes appear to be under good control at lower energies, where there are numerous polarization data, even though there are not enough types of polarization data to uniquely determine the amplitudes [44]. The disagreement at higher energies is not surprising given the fact that these are the first results for polarization transfer for this reaction; the phase shift analysis is not sufficiently constrained by the existing data set.

The longitudinal in-plane polarization transfer $C_{z^{\prime}}$, as shown in Figs. 13 and 14, does not show large polarizations as predicted from Ref. [17]. Also $C_{z^{\prime}}$ does not appear to reach a constant value at each angle, as predicted by pQCD

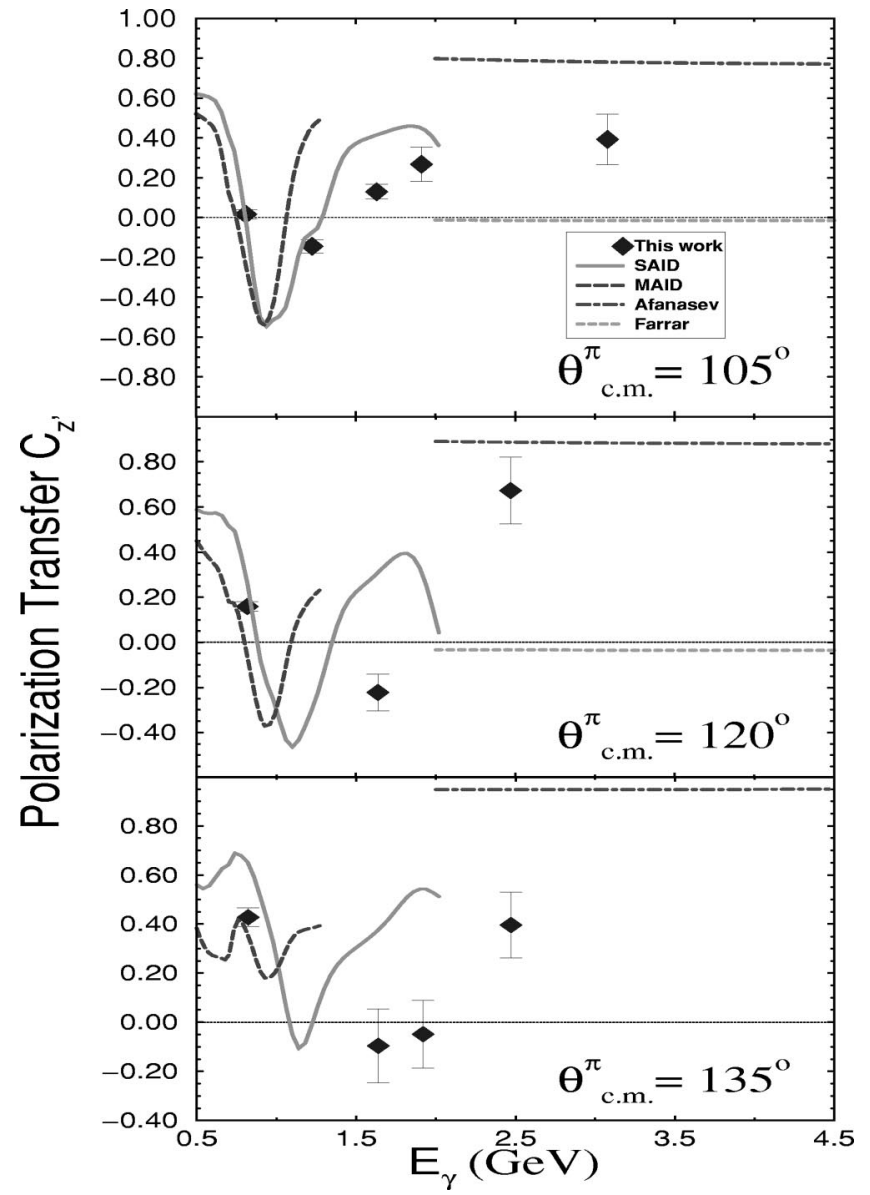

FIG. 14. Top to bottom: Polarization transfer $C_{z^{\prime}}$ in neutral pion photoproduction at $\theta_{\text {c.m. }}=105^{\circ}, 120^{\circ}, 135^{\circ}$. Only statistical uncertainties are shown. The four curves SAID [22], MAID [23], Afanasev [17], and Farrar [16] are described in the text. All data and calculations are in the lab frame defined in Sec. I.

(Ref. [16]), however the $p_{T}$ values for $C_{z^{\prime}}$ are well below the scaling threshold of $1.3 \mathrm{GeV} / c$. The phase shift analysis curves from SAID and MAID tend to agree better with the data at lower energies, and diverge with increasing energy, as was also the case for $C_{x^{\prime}}$.

No $C_{x^{\prime}}$ and $C_{z^{\prime}}$ data are shown at $E_{\gamma}=1.1 \mathrm{GeV}$ in Table II, because an unpolarized electron beam was used at this energy. For certain other kinematic settings no $p_{y}$ or $C_{z^{\prime}}$ data are given, due to the unfavorable spin transport that makes the uncertainty $\geqslant 0.3$. The data points recorded with an unpolarized beam at $E_{\gamma}=1.1 \mathrm{GeV}$ are consistent with zero, illustrating the quality of the data.

Figures 15 and 16 show the angular distributions for the induced polarization at several photon energies. Note that the data in each figure were taken over a range of energies.

Polarization measurements provide a powerful method for studying nucleon structure. The vector polarization $p_{y}$ is related to the differential cross section $d \sigma / d \Omega$ and the differential polarization $d p / d \Omega$ by the expression

$$
p_{y}=\frac{1}{j} \frac{d p / d \Omega}{d \sigma / d \Omega},
$$




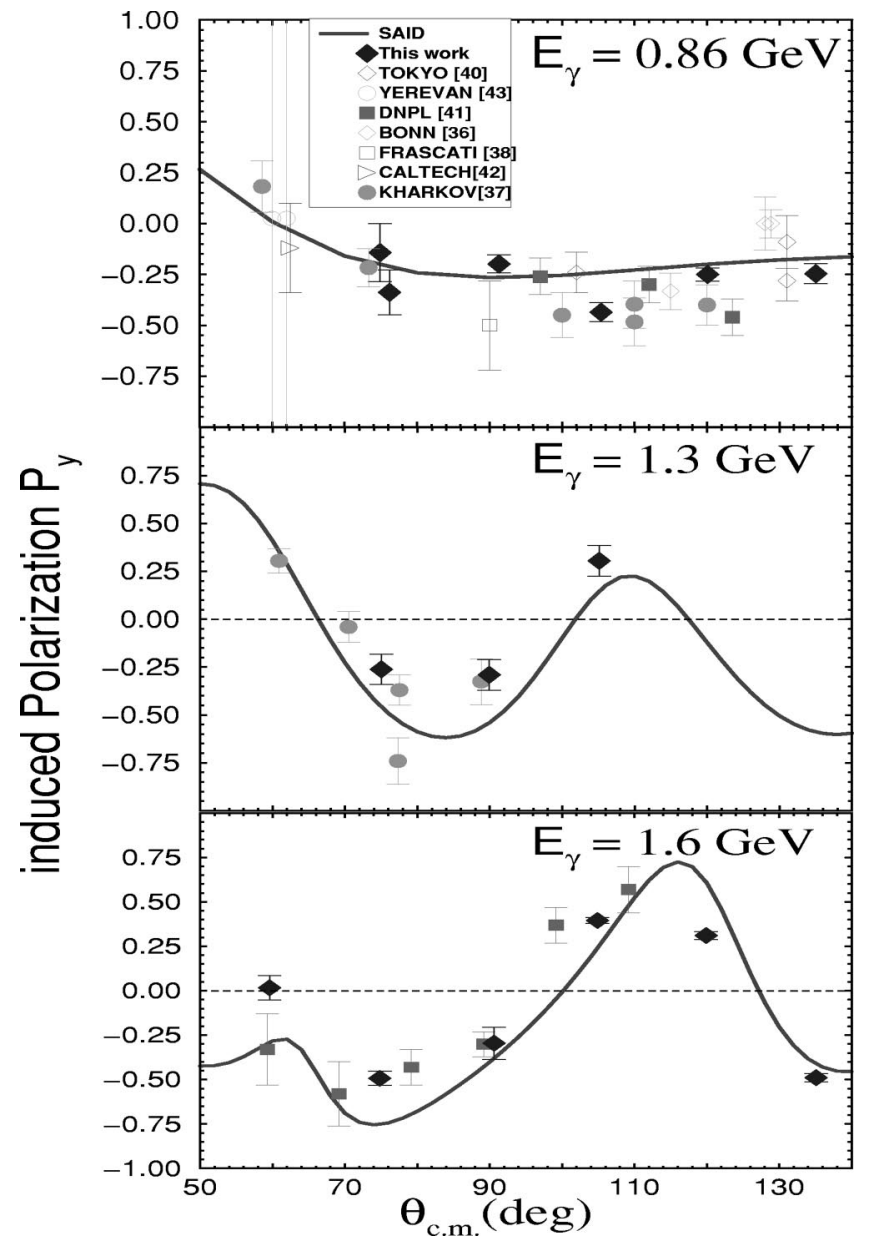

FIG. 15. Top to bottom: Angular distributions of induced polarization $p_{y}$ in neutral pion photoproduction at $E_{\gamma}=0.86 \mathrm{GeV}, 1.3$ $\mathrm{GeV}$, and $1.6 \mathrm{GeV}$. The curve SAID [22] shown here is described in the text. Note that the JLab data were at energies of $0.8,1.2$, and 1.6 $\mathrm{GeV}$ as given in Table II.

where $j$ is the spin of the emitted particle. The differential polarization can be written as

$$
\frac{d p}{d \Omega}=\sum_{\nu=1} B_{\nu} P_{\nu}^{1}(\cos \theta)
$$

where the $B_{\nu}$ contain the reaction matrix elements as discussed in Ref. [45]. Assuming two amplitudes, $\nu$ ranges from 1 to the sum of the angular momentum of the two amplitudes that produce the interference effect giving rise to the polarization. From the nature of the first associated Legendre polynomial, the angular distribution of the differential polarization will behave essentially like $\sin (\nu \theta)$. For example, if an $s$ wave in the outgoing $\pi N$ system interferes with a $d$ wave, then a $\sin (2 \theta)$ dependence would be expected. The angular dependence for $p_{y}$ at $2.5 \mathrm{GeV}$ suggests a strong oscillatory behavior, the $\sin (12 \theta)$ curve indicates very high partial waves. This suggests that the background or resonances contain relatively high partial waves. It is apparent that much more finely binned polarization angular distributions will be needed to understand these high-energy reso-

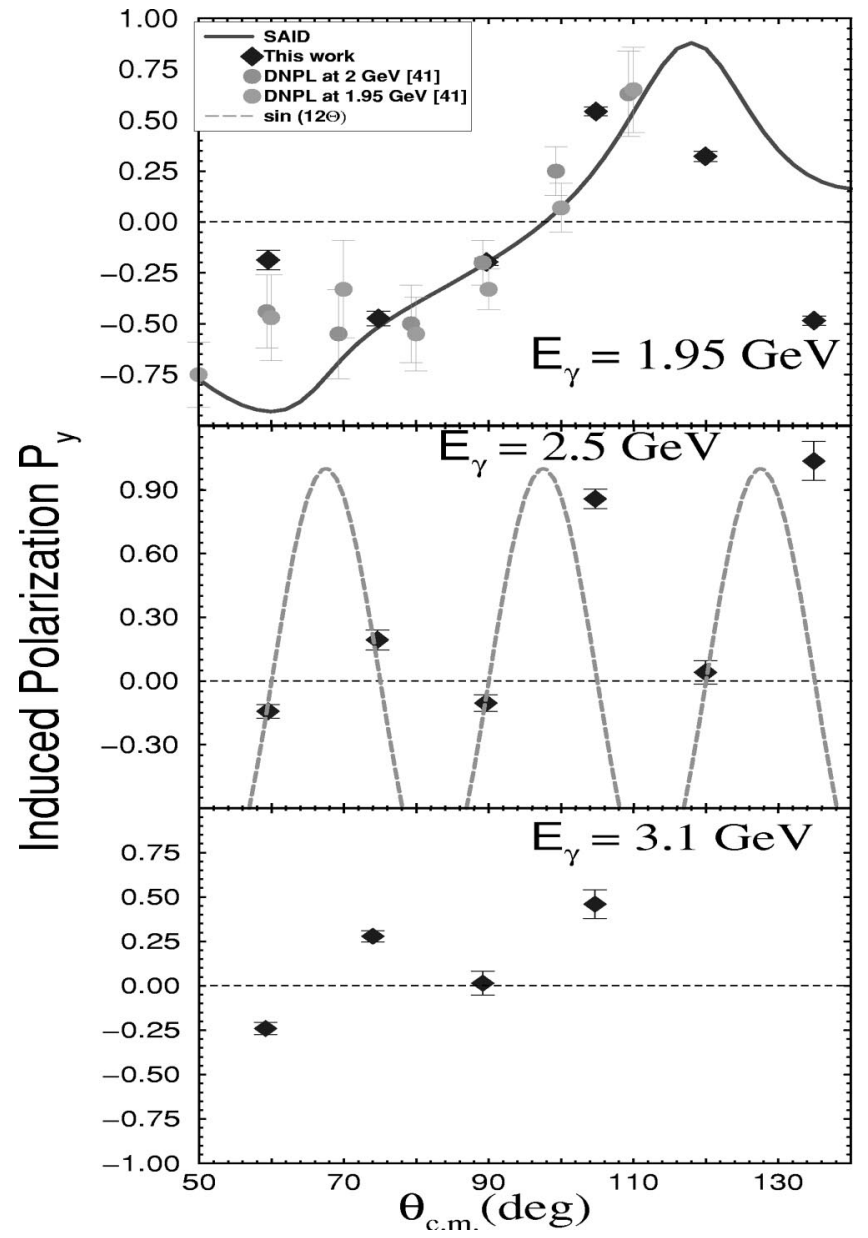

FIG. 16. Top to bottom: Angular distributions of induced polarization $p_{y}$ in neutral pion photoproduction at $E_{\gamma}=1.9 \mathrm{GeV}, 2.5$ $\mathrm{GeV}$, and $3.1 \mathrm{GeV}$. The curve SAID [22] shown here is described in the text. The $\sin (12 \theta)$ curve at $2.5 \mathrm{GeV}$ is drawn merely to illustrate the strong angular dependence. Note that the JLab data were at energies of $1.9,2.5$, and $3.1 \mathrm{GeV}$ as given in Table II.

nance contributions in detail. The strong angular dependence appears to persist up to $3.1 \mathrm{GeV}$ as shown by the lower panel in Fig. 15. This suggests that even at $E_{\gamma}=3.1 \mathrm{GeV}$ ( $W$ $=2.6 \mathrm{GeV}$ ), resonances are needed to explain the data, and quark models that sum over the resonances will be unable to reproduce the polarizations.

\section{CONCLUSIONS}

Present induced polarization data extend the world data set for $\pi^{0}$ photoproduction from the proton to much higher photon energies at large angle. In the overlap region, induced polarization data agree well with existing data, and also with the phase shift analysis models: SAID and MAID. This work also presents the first data set in polarization transfer observables $C_{x^{\prime}}$ and $C_{z^{\prime}}$ for the ${ }^{1} H(\vec{\gamma}, \vec{p}) \pi^{0}$ reaction. The nonzero nature of $p_{y}$ and the polarization transfer component, $C_{x^{\prime} \text { c.m. }}$, shows that hadron helicity is not conserved. Therefore, pQCD cannot simply explain the data up to a photon energy of $3.1 \mathrm{GeV}$ or a $p_{T}$ of $1.1 \mathrm{GeV} / c$ for ${ }^{1} H(\vec{\gamma}, \vec{p}) \pi^{0}$ 
reaction. Furthermore, the strong angle-dependent nature of the induced polarization $p_{y}$ might be an indication of interference between resonances and the nonresonant background. This confirms the importance of polarization observables as a powerful tool to look for resonance effects. Since the angular distributions of $p_{y}$ at $2.5 \mathrm{GeV}$ and $3.1 \mathrm{GeV}$ have strong oscillations, a data set finely binned in angle would be extremely useful in advancing the theoretical analysis.

The data do not agree with any of the existing quark model calculations; further there is no support in these data for the general prediction that the polarizations should become energy independent. Failure of the Afanasev prediction implies that the data do not favor factorization at relatively low values of $p_{T}$. While theoretical predictions for the polarization observables in ${ }^{1} H(\vec{\gamma}, \vec{p}) \pi^{0}$ reaction are very limited and highly desirable, this data set, rich in both angular and energy bins, will help develop a better understanding of photo-pion production in the $\mathrm{GeV}$ region.

\section{ACKNOWLEDGMENTS}

We are particularly indebted to Dick Arndt, Igor Strakovsky, and Ron Workman for many discussions concerning SAID and the polarization observables in the c.m. and lab frames. We thank G. Farrar, N. Isgur, T.-S.H. Lee, A. Radyushkin, and J. Ralston for many interesting discussions. We thank the JLab physics and accelerator divisions for their support, especially Ed Folts and the Hall A technical staff, and Charles Sinclair and the polarized source group. This work was supported by the U.S. Department of Energy, the U.S. National Science Foundation, the Natural Sciences and Engineering Research Council of Canada, the French Commissariat à l'Energie Atomique and Center National de la Recherche Scientifique, the Italian National Institute for Nuclear Physics, and the Swedish Natural Science Research Council. The Southeastern Universities Research Association (SURA) operates the Thomas Jefferson National Accelerator Facility under DOE Contract No. DE-AC05-84ER40150. The polarimeter was funded by the U.S. National Science Foundation, Grant Nos. PHY 9213864 and PHY 9213869. The Møller polarimeter was funded by Grant No. INTAS-99125 .

\section{APPENDIX: SPIN TRANSFORMATION FROM THE LABORATORY FRAME TO CENTER-OF-MASS COORDINATES}

The transformation of the proton spin from lab to centerof-mass frames is performed by conventional Lorentz boosts and rotations. We detail our procedures here since the transformation appears to be unfamiliar to most readers; a more advanced presentation can be found in Dmitrašinović [46].

The proton spin can be represented by a four-vector

$$
S_{\mu}=\left(S_{0} S_{x} S_{y} S_{z}\right)
$$

The lab to center-of-mass conversion then involves boosts of the form

$$
\left(\begin{array}{c}
S_{0^{\prime}} \\
S_{x^{\prime}} \\
S_{y^{\prime}} \\
S_{z^{\prime}}
\end{array}\right)=\left(\begin{array}{cccc}
\gamma & 0 & 0 & -\beta \gamma \\
0 & 1 & 0 & 0 \\
0 & 0 & 1 & 0 \\
-\beta \gamma & 0 & 0 & \gamma
\end{array}\right)\left(\begin{array}{c}
S_{0} \\
S_{x} \\
S_{y} \\
S_{z}
\end{array}\right),
$$

and rotations of the form

$$
\left(\begin{array}{c}
S_{0^{\prime}} \\
S_{x^{\prime}} \\
S_{y^{\prime}} \\
S_{z^{\prime}}
\end{array}\right)=\left(\begin{array}{cccc}
1 & 0 & 0 & 0 \\
0 & \cos \theta & 0 & \sin \theta \\
0 & 0 & 1 & 0 \\
0 & -\sin \theta & 0 & \cos \theta
\end{array}\right)\left(\begin{array}{c}
S_{0} \\
S_{x} \\
S_{y} \\
S_{z}
\end{array}\right) .
$$

The proton kinematics in the lab (center-of-mass) frame are given by the factors $\gamma_{l a b}, \beta_{l a b}, \theta_{l a b}\left(\gamma_{\text {c.m. }}, \beta_{\text {c.m. }}, \theta_{\text {c.m. }}\right)$, with the usual definitions, while the lab to center-of-mass transformation involves a boost with $\gamma_{\mathrm{c} . \mathrm{m} . \mathrm{l}}$ and $\beta_{\mathrm{c} . \mathrm{m} . \mathrm{l}}$. It is possible to reduce these eight parameters to various choices of three independent ones, such as $\gamma_{l a b}, \theta_{l a b}$, and $\gamma_{\text {c.m.l. }}$.

The proton polarimeter measures the transverse components of the spin in the spectrometer focal plane; the transverse components are Lorentz invariants. These two-spin components are then used to determine the three-vector spin components at the target; this is possible since the $\hat{y}$-spin component at the target is helicity independent, while the $\hat{x}$ and $\hat{z}$ components are helicity dependent. Although the longitudinal component is frame dependent, it is conventional to quote the rest frame value. The lab (or c.m.) frame is used only to determine the direction of the axes. This convention results in a fixed normalization for the proton spin vector, $\Sigma_{i=1,3} S_{i}^{2}=1$, which is simpler than having to calculate the four-vector $S_{\mu} S^{\mu}=-1$. The transformation from lab to c.m. frames then involves a boost from the scattered lab-oriented rest frame to the scattered lab frame

$$
\left(\begin{array}{cccc}
\gamma_{l a b} & 0 & 0 & \beta_{l a b} \gamma_{l a b} \\
0 & 1 & 0 & 0 \\
0 & 0 & 1 & 0 \\
\beta_{l a b} \gamma_{l a b} & 0 & 0 & \gamma_{l a b}
\end{array}\right)
$$

a rotation to the lab frame (oriented along the incident photon direction)

$$
\left(\begin{array}{cccc}
1 & 0 & 0 & 0 \\
0 & \cos \theta_{l a b} & 0 & \sin \theta_{l a b} \\
0 & 0 & 1 & 0 \\
0 & -\sin \theta_{l a b} & 0 & \cos \theta_{l a b}
\end{array}\right)
$$

a boost to the c.m. frame

$$
\left(\begin{array}{cccc}
\gamma_{\text {c.m.l }} & 0 & 0 & -\beta_{\text {c.m.l }} \gamma_{\text {c.m.l }} \\
0 & 1 & 0 & 0 \\
0 & 0 & 1 & 0 \\
-\beta_{\text {c.m.l }} \gamma_{\text {c.m.l }} & 0 & 0 & \gamma_{\text {c.m.l }}
\end{array}\right),
$$


a rotation to the scattered c.m. frame

$$
\left(\begin{array}{cccc}
1 & 0 & 0 & 0 \\
0 & \cos \theta_{\text {c.m. }} & 0 & -\sin \theta_{\text {c.m. }} \\
0 & 0 & 1 & 0 \\
0 & \sin \theta_{\text {c.m. }} & 0 & \cos \theta_{\text {c.m. }}
\end{array}\right)
$$

and a boost to the scattered c.m.-oriented rest frame.

$$
\left(\begin{array}{cccc}
\gamma_{\text {c.m. }} & 0 & 0 & -\beta_{\text {c.m. }} \gamma_{\mathrm{c.m} .} \\
0 & 1 & 0 & 0 \\
0 & 0 & 1 & 0 \\
-\beta_{\text {c.m. }} \gamma_{\text {c.m. }} & 0 & 0 & \gamma_{\text {c.m. }}
\end{array}\right) .
$$

The product of these five arrays leads to a transformation that is purely a rotation that mixes the in-plane, $x$ and $z$, spin components, as illustrated in Fig. 8,

$$
\left(\begin{array}{l}
S_{0 \text { c.m. }} \\
S_{x \text { c.m. }} \\
S_{y \text { c.m. }} \\
S_{z_{\text {c c.m. }}}
\end{array}\right)=\left(\begin{array}{cccc}
1 & 0 & 0 & 0 \\
0 & \cos \theta_{\text {rot }} & 0 & -\sin \theta_{\text {rot }} \\
0 & 0 & 1 & 0 \\
0 & \sin \theta_{\text {rot }} & 0 & \cos \theta_{\text {rot }}
\end{array}\right)\left(\begin{array}{c}
S_{0 l a b} \\
S_{x l a b} \\
S_{y_{l a b}} \\
S_{z_{l a b}}
\end{array}\right) .
$$

We choose $\beta$ and $\theta$ always positive, which makes some of the signs opposite the usual convention given above in Eqs.
(A2) and (A3). The expressions that result in terms of the eight kinematic parameters from multiplying together the five components of the transformation are, without simplification, not obviously in agreement with the result given here; the equivalence is easily checked numerically. The diagonal matrix elements are given by

$$
\begin{aligned}
& \cos \theta_{\text {rot }}=\cos \theta_{\text {lab }} \cos \theta_{\text {c.m. }}+\gamma_{\text {c.m. } .} \sin \theta_{\text {lab }} \sin \theta_{\text {c.m. }} \\
& =\gamma_{\text {c.m. }} \gamma_{l a b}\left[\sin \theta_{\text {lab }} \sin \theta_{\text {c.m. }}+\gamma_{\text {c.m.l }} \cos \theta_{\text {c.m. }}\right. \\
& \times\left(\cos \theta_{l a b}-\beta_{\text {c.m. } .} \beta_{l a b}\right) \\
& \left.-\beta_{\text {c.m. }} \gamma_{\text {c.m.l }}\left(\beta_{l a b}-\beta_{\text {c.m.l }} \cos \theta_{l a b}\right)\right] \text {, }
\end{aligned}
$$

while the off-diagonal matrix elements are given by

$$
\begin{aligned}
& \sin \theta_{r o t}=\gamma_{l a b}\left[-\sin \theta_{l a b} \cos \theta_{\text {c.m. }}\right. \\
& \left.+\gamma_{\text {c.m.l } 1} \sin \theta_{\text {c.m. }}\left(\cos \theta_{\text {lab }}-\beta_{\text {c.m.1. }} \beta_{\text {lab }}\right)\right] \\
& =\gamma_{\mathrm{c} . \mathrm{m} .}\left[\cos \theta_{\text {lab }} \sin \theta_{\mathrm{c} . \mathrm{m} .}-\gamma_{\mathrm{c} . \mathrm{m} . \mathrm{l}} \sin \theta_{\text {lab }} \cos \theta_{\mathrm{c} . \mathrm{m} \text {. }}\right. \\
& \left.-\beta_{\text {c.m. }} \beta_{\text {c.m.l }} \gamma_{\text {c.m.l }} \sin \theta_{\text {lab }}\right] \text {. }
\end{aligned}
$$

The transformation described above, converts from the lab to c.m. coordinate system. By changing the sign of the $\sin \theta_{\text {rot }}$ terms in Eq. (A9), one can transform instead from the c.m. to the lab coordinate system, as was done in this analysis.
[1] Data and references can be found in D. Menze, W. Pfeil, and R. Wilcke, Compilation of Pion Photoproduction Data (Physikalisches Institut der Universitat, Bonn, 1977).

[2] S.J. Brodsky and G.R. Farrar, Phys. Rev. Lett. 31, 1153 (1973); V. Matveev et al., Lett. Nuovo Cimento Soc. Ital. Fis. 7, 719 (1973).

[3] R.L. Anderson et al., Phys. Rev. D 14, 679 (1976).

[4] C. Bochna et al., Phys. Rev. Lett. 81, 4576 (1998).

[5] J.E. Belz et al., Phys. Rev. Lett. 74, 646 (1995).

[6] S.J. Freedman et al., Phys. Rev. C 48, 1864 (1993).

[7] J. Napolitano et al., Phys. Rev. Lett. 61, 2530 (1988).

[8] E.C. Schulte et al., Phys. Rev. Lett. 87, 102302 (2001).

[9] See S.J. Brodsky and G.P. Lepage, Phys. Rev. D 24, 2848 (1981), and references therein.

[10] D.G. Crabb et al., Phys. Rev. Lett. 65, 3241 (1990).

[11] P.V. Landshoff, Phys. Rev. D 10, 1024 (1974).

[12] T. Gousset, B. Pire, and J.P. Ralston, Phys. Rev. D 53, 1202 (1996).

[13] C. Carlson and M. Chachkhunashvili, Phys. Rev. D 45, 2555 (1992).

[14] A. Afanasev, C. Carlson, and C. Wahlquist, Phys. Rev. D 61, 034014 (2000).

[15] G.A. Miller, M.R. Frank, nucl-th/0201021.

[16] G.R. Farrar, K. Huleihel, and H. Zhang, Nucl. Phys. B349, 655 (1991).

[17] A. Afanasev, C. Carlson, and C. Wahlquist, Phys. Lett. B 398, 393 (1997)
[18] M. Guidal, J.-M. Laget, and M. Vanderhaeghen, Nucl. Phys. A627, 645 (1997).

[19] K. Wijesooriya et al., Phys. Rev. Lett. 86, 2975 (2001).

[20] H. Olsen and L.C. Maximon, Phys. Rev. 114, 887 (1959).

[21] I.S. Barker, A. Donnachie, and J.K. Storrow, Nucl. Phys. B95, 347 (1975).

[22] R.A. Arndt et al., Phys. Rev. C 53, 430 (1996); 56, 577 (1997).

[23] D. Drechsel et al., Nucl. Phys. A645, 145 (1999); version MAID 2000.

[24] A.V. Afanasev, I. Akushevich, A. Ilyichev, and N.P. Merenkov Phys. Lett. B 514, 369 (2001).

[25] M.W. McNaughton et al., Nucl. Instrum. Methods Phys. Res. A 241, 435 (1985).

[26] M.K. Jones et al., Phys. Rev. Lett. 84, 1398 (2000); V. Punjabi et al. (unpublished).

[27] K. Wijesooriya et al. (unpublished).

[28] O. Gayou et al., Phys. Rev. C 64, 038202 (2001).

[29] N.E. Cheung et al., Nucl. Instrum. Methods Phys. Res. A 363, 561 (1995); B. Bonin et al., ibid. 288, 379 (1990).

[30] K. Makino and M. Berz, COSY INFINITY version 8, Nucl. Instrum. Methods Phys. Res. A 427, 338 (1999).

[31] D. Dutta, Phys. Rev. C 65, 044619 (2002).

[32] David A. Jenkins and Igor I. Strakovsky, Phys. Rev. C 52, 3499 (1995).

[33] A.V. Radyushkin, Phys. Rev. D 58, 11408 (1998); M. Diehl, Eur. Phys. J. C 8, 409 (1999); H.W. Huang and P. Kroll, ibid. 17, 423 (2000). 
[34] S. Capstick, Phys. Rev. D 46, 2864 (1992).

[35] S. Capstick and W. Roberts, Phys. Rev. D 49, 4570 (1994).

[36] P. Blüm et al., preprint Bonn PI1-105 70; Z. Phys. A 277, 311 (1976); D. Trines et al., PRP PI1-160 72.

[37] A.I. Derebchinski et al., JETP Lett. 43, 218 (1976); A.A. Zybalov et al., Sov. J. Nucl. Phys. 28, 52 (1978); JETP Lett. 37, 205 (1973); V.G. Gorbenko et al., Sov. J. Nucl. Phys. 26, 167 (1977); A.I. Derebchinski et al., JETP Lett. 39, 30 (1974); A.S. Bratashevski et al., Nucl. Phys. B166, 525 (1980); Sov. J. Nucl. Phys. 33, 538 (1981); 35, 33 (1982); 38, 233 (1983); 41, 960 (1985); 42, 417 (1985); 46, 635 (1987).

[38] R. Querzoli et al., Nuovo Cimento Lett. 19, 53 (1961); L. Bertanza et al., ibid. 24, 734 (1962); C. Mencuccini et al., Phys. Rev. 126, 1181 (1962).

[39] J.O. Maloy et al., Phys. Rev. 139, B733 (1965); D.E. Lundquist et al., Phys. Rev. 168, 1527 (1968).
[40] S. Hayakawa et al., J. Phys. Soc. Jpn. 25, 307 (1968); S. Kabe et al., Nucl. Phys. B50, 17 (1972); T. Kato et al., ibid. B168, 1 (1980).

[41] P.J. Bussey et al., Nucl. Phys. B154, 492 (1979).

[42] E.D. Bloom et al., Phys. Rev. Lett. 19, 671 (1967).

[43] R.O. Avakyan et al., Sov. J. Nucl. Phys. 37, 199 (1983); 46, 853 (1987); 48, 1030 (1988); 53, 448 (1991); K.Sh. Agababyan et al., ibid. 50, 834 (1989); M.M. Asaturyan et al., JETP Lett. 44, 341 (1986).

[44] W.-T. Chiang and F. Tabakin, Phys. Rev. C 55, 2054 (1997).

[45] R.M. Laszewski and R.J. Holt, At. Data Nucl. Data Tables 19, 305 (1977); J.E. Monahan et al., ibid. 19, 97 (1977); J.E. Monahan et al., Phys. Rev. C 20, 389 (1979).

[46] V. Dmitrašinović, Phys. Rev. C 47, 2195 (1993). Note that this paper gives formulas for the spin precession angle, $\omega$, related to the rotation angle by $\theta_{\text {rot }}=\theta_{\text {c.m. }}-\theta_{\text {lab }}-\omega$. 